Linköping Studies in Science and Technology

Dissertations, No. 1926

\title{
Learning Convolution Operators for Visual Tracking
}

\author{
Martin Danelljan
}


Edition 1:1

(C) Martin Danelljan, 2018

ISBN 978-91-7685-332-0

ISSN 0345-7524

URL http://urn.kb.se/resolve?urn=urn:nbn:se:liu:diva-147543

Published articles have been reprinted with permission from the respective copyright holder.

Typeset using $\mathrm{X}_{\mathrm{H}} \mathrm{T}_{\mathrm{E}} \mathrm{X}$

Printed by LiU-Tryck, Linköping 2018 


\section{POPULÄRVETENSKAPLIG SAMMANFATTNING}

Visuell tracking är ett grundläggande forskningsområde inom fältet datorseende. Det är en viktig del i många existerande och kommande tekniker, inklusive robotik, självkörande bilar, augmented reality, och 3D-rekonstruktion. Visuell tracking syftar till att automatiskt spåra en bildregion eller visuellt objekt $\mathrm{i}$ en sekvens av bilder. Det spårade målet utgörs av till exempel ett ansikte, en bil eller ett distinkt landmärke. Vi människor klarar av att spåra visuella mål utan ansträngning som en del av vårt vardagliga beteende. Men eftersom detta har visat sig vara svårt att automatisera, är visuell tracking ett fortsatt aktivt forskningsområde.

Många trackingmetoder är utvecklade för en viss tilltänkt applikation genom att först begränsa problemet. Målet kan till exempel antas vara en viss typ av objekt, eller att kameran antas vara statisk. I generell visuell tracking gör man däremot inga sådana antaganden, vilket försvårar problemet men samtidigt ökar applicerbarheten. Eftersom ingen information om målet är givet, undantaget dess initiala position, måste trackingmetoden anpassa en modell av målets utseende under själva spårningen. Detta är en typ av maskininlärningsproblem, vilket behandlas i denna avhandling.

Huvudsyftet med denna avhandling är att studera och utveckla en särskild klass av metoder, som kallas diskriminativa korrelationsfilter (Discriminative Correlation Filter, DCF). Dessa metoder har visat sig särskilt lämpade för visuell tracking. De använder sig av egenskaper hos fouriertransformen för att effektivt träna ett korrelationsfilter. Inlärningen av filtret sker diskriminativt, genom att minimera en kvadratisk felfunktion. Det resulterande filtret kan sedan appliceras på en ny bild för att lokalisera målet.

Det huvudsakliga bidraget av avhandlingen är att utveckla maskininlärningsmetoden som används i DCF-ramverket, med syftet att förbättra robustheten och noggrannheten av spårningen. Ett antal väsentliga förbättringar föreslås och analyseras. Nya effektiva metoder för att uppdatera modellen av målet presenteras. Vidare införs en spatiell regulariseringskomponent för att adressera de negativa aspekterna av cirkulär faltning. En annan svårighet uppkommer av att trackingmetoden själv måste annotera nya träningsexempel för uppdateringen av modellen. Det kan leda till felannoteringar eller att korrumperad data inkluderas i träningsmängden. I avhandlingen utvecklas en metod för att reducera effekten av sådan träningsdata genom att adaptivt vikta om datasetet. Dessutom presenteras en kontinuerlig formulering av maskininlärningsproblemet, vilket möjliggör integrering av särdrag med olika upplösning och subpixelprecis spårning av målet. Slutligen, undersöks metoder för att minska beräkningskostnaden.

Det andra bidraget av avhandlingen är att undersöka olika typer av särdrag för visuell tracking. Särdrag är en representation av bilden, som i trackingfallet syftar till att förbättra förmågan att särskilja mellan målets och bakgrundens utseende. En genomgående analys av olika färgsärdrag görs. Dessutom undersöks djupa särdrag, vilket under de senaste åren har visat sig mycket användbara inom datorseende. Undersökningen visar på att både tidiga och djupa särdrag från ett faltningsnätverk bidrar till förbättrad spårningsprestanda.

Inom de flesta användningsområden av visuell tracking är det viktigt att utöver målets position även spåra dess storlek, eftersom denna är relaterad till avståndet mellan målet och kameran. Som det tredje större bidraget av avhandlingen undersöks därför olika metoder för att skatta målets storlek. Metoden som presenteras bygger på ett endimensionellt storleksfilter, som noggrant och effektivt kan beräkna storleken av målet. 


\begin{abstract}
Visual tracking is one of the fundamental problems in computer vision. Its numerous applications include robotics, autonomous driving, augmented reality and 3D reconstruction. In essence, visual tracking can be described as the problem of estimating the trajectory of a target in a sequence of images. The target can be any image region or object of interest. While humans excel at this task, requiring little effort to perform accurate and robust visual tracking, it has proven difficult to automate. It has therefore remained one of the most active research topics in computer vision.
\end{abstract}

In its most general form, no prior knowledge about the object of interest or environment is given, except for the initial target location. This general form of tracking is known as generic visual tracking. The unconstrained nature of this problem makes it particularly difficult, yet applicable to a wider range of scenarios. As no prior knowledge is given, the tracker must learn an appearance model of the target on-the-fly. Cast as a machine learning problem, it imposes several major challenges which are addressed in this thesis.

The main purpose of this thesis is the study and advancement of the, so called, Discriminative Correlation Filter (DCF) framework, as it has shown to be particularly suitable for the tracking application. By utilizing properties of the Fourier transform, a correlation filter is discriminatively learned by efficiently minimizing a least-squares objective. The resulting filter is then applied to a new image in order to estimate the target location.

This thesis contributes to the advancement of the DCF methodology in several aspects. The main contribution regards the learning of the appearance model: First, the problem of updating the appearance model with new training samples is covered. Efficient update rules and numerical solvers are investigated for this task. Second, the periodic assumption induced by the circular convolution in DCF is countered by proposing a spatial regularization component. Third, an adaptive model of the training set is proposed to alleviate the impact of corrupted or mislabeled training samples. Fourth, a continuousspace formulation of the DCF is introduced, enabling the fusion of multiresolution features and sub-pixel accurate predictions. Finally, the problems of computational complexity and overfitting are addressed by investigating dimensionality reduction techniques.

As a second contribution, different feature representations for tracking are investigated. A particular focus is put on the analysis of color features, which had been largely overlooked in prior tracking research. This thesis also studies the use of deep features in DCF-based tracking. While many vision problems have greatly benefited from the advent of deep learning, it has proven difficult to harvest the power of such representations for tracking. In this thesis it is shown that both shallow and deep layers contribute positively. Furthermore, the problem of fusing their complementary properties is investigated.

The final major contribution of this thesis regards the prediction of the target scale. In many applications, it is essential to track the scale, or size, of the target since it is strongly related to the relative distance. A thorough analysis of how to integrate scale estimation into the DCF framework is performed. A one-dimensional scale filter is proposed, enabling efficient and accurate scale estimation. 


\section{Acknowledgments}

My time as a PhD student has been an amazing journey. It is a journey that I have been fortunate to share with many friends and colleagues, whose importance cannot be overstated. Much thanks to them, all the hard work, late nights, and stressful deadlines, have always been fun. I have been working in some great teams, where we have approached the challenge together. Particularly memorable are the times when the focus and intensity were at its peak. But also, when we shared the success or enjoyed a nice conference together.

I am grateful to all colleagues at the Computer Vision Lab at Linköping University for all the help, support, interesting discussions and collaborations. I particularly want to thank my main supervisor, Michael Felsberg. He has always been supportive and available for discussion. And his advice has been of much value throughout this journey. Not to forget, Michael gave me the freedom to pursue the research questions and ideas that I found of interest.

I have found it fun and inspiring to cooperate with other $\mathrm{PhD}$ students. And most papers included in this thesis are fruits of such collaborations, for which I am very thankful. Gustav Häger worked with me on tracking, most intensely during 2014 and 2015. Andreas Robinson, with whom I shared office and many insightful discussions, also collaborated with me during the ECCV 2016 period. Joakim Johnander, who like Gustav started as a master's thesis student of mine and moved on to start a $\mathrm{PhD}$, has cooperated with me on tracking ideas since 2017. I also have much to thank Goutam Bhat, who while currently finishing his master's with finest grades, has continued to work closely with me on research and other endeavors.

While this thesis is primarily about visual tracking, I have also spent significant effort on other lines of research. Giulia Meneghetti worked with me on point cloud registration during 2015 and early 2016. In collaboration with Felix Järemo Lawin, this line of research has continued with new and exciting ideas. I have also been fortunate to work together with Per-Erik Forssèn and Klas Nordberg on some of these projects.

It is not easy to find the correct words to describe the importance of the collaboration with my co-supervisor, Fahad Khan. Over these years, we have had such a close cooperation. Discussing ideas, papers, planning. But most 
importantly, the writing. The hours we have spent, often very late, polishing individual words on CVPR, ICCV and ECCV submissions. And somehow, it was always fun and rewarding. Fahad has been an enormous support, and his mood always positive. Cheering up even during the hardest struggles. I cannot help to remember a particular time, the summer of 2015. I was on a Beneteau Oceanis 46 anchored in beautiful Karlskrona when some, in our view, very unfair reviews of our paper arrived. Fahad beeing in Pakistan at the time. We had one week to write an incredibly tight rebuttal to convince the reviewers otherwise. On Fahad's positive note, that we can do this, we got to work. Imagine, several hour long skype calls between a $4 \mathrm{G}$ connection from a sailboat on the southern coast of Sweden and a shaky Pakistani broadband, a few days in a row. Carefully carving out every single word of the rebuttal. And thanks to this, the paper got in.

Lastly, I want to thank my beloved family for the continued support. My mother Alice Danelljan, father Jan Mårtensson, and my sister Marielle Danelljan.

Martin Danelljan

Linköping

May 2018 


\section{Contents}

Abstract

Acknowledgments vi

Contents vii

I Background 1

1 Introduction $\quad 3$

1.1 Visual Tracking . . . . . . . . . . . . . . . 3

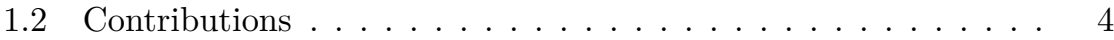

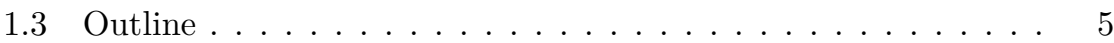

1.4 Included Publications . . . . . . . . . . . . . . . . . . . . 6

1.5 Additional Publications . . . . . . . . . . . . . . 15

2 From Lucas-Kanade to MOSSE $\quad 17$

2.1 A Generative Tracking Model . . . . . . . . . . . . . . . . . . . . . . 17

2.2 Incorporating Background Information . . . . . . . . . . . . . . 20

2.3 Generative versus Discriminative Approaches . . . . . . . . . . . . . . 22

2.4 A Discriminative Tracking Model . . . . . . . . . . . . . . . . 23

2.5 Linear Regression . . . . . . . . . . . . . . . . . . . . 24

2.6 A Bayesian Perspective on Linear Regression . . . . . . . . . . . . . . 26

2.7 Convolution and the Fourier Transform . . . . . . . . . . . . . 27

2.8 The MOSSE Tracker . . . . . . . . . . . . . . . . . . . 29

3 Discriminative Correlation Filters 33

3.1 The Kernelized DCF . . . . . . . . . . . . . . . . . 33

3.2 Multidimensional Feature Maps . . . . . . . . . . . . . . . . 34

3.3 Scale Estimation . . . . . . . . . . . . . . . . . . . 36

3.4 Periodic Assumption and Spatial Regularization . . . . . . . . . 38

3.5 Adaptive Training Set Management . . . . . . . . . . . . . . . . . . . 41

3.6 Continuous Formulation . . . . . . . . . . . . . . . . . . . . . 42

3.7 Dimensionality Reduction . . . . . . . . . . . . . . . . . . 44 


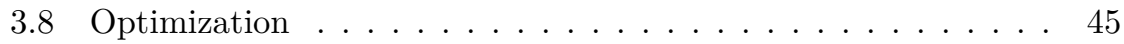

$\begin{array}{llr}4 & \text { Image Features } & 49\end{array}$

4.1 Invariance and Discriminative Power . . . . . . . . . . . . . 49

4.2 Color Features . . . . . . . . . . . . . . . . . . 51

4.3 Histogram of Oriented Gradients . . . . . . . . . . . . . . . . . 52

4.4 Deep Features . . . . . . . . . . . . . . . . . . . 53

4.5 Deep Motion Features . . . . . . . . . . . . . . . . . . . 54

5 Concluding Remarks $\quad 57$

$\begin{array}{ll}\text { Bibliography } & 61\end{array}$

$\begin{array}{lr}\text { II Publications } & 71\end{array}$

Paper A $\begin{aligned} & \text { Adaptive Color Attributes for Real-Time Visual } \\ & \text { Tracking }\end{aligned}$

$\begin{array}{lllllll}\text { Paper B } & \begin{array}{l}\text { Coloring } \\ \text { Tracking }\end{array} & \text { Channel } & \text { Representations } & \text { for } & \text { Visual } & 87\end{array}$

$\begin{array}{lll}\text { Paper C Discriminative Scale Space Tracking } & 103\end{array}$

Paper D Learning Spatially Regularized Correlation Filters 121

$\begin{array}{ll}\text { Paper E } & \begin{array}{l}\text { Convolutional Features for Correlation Filter Based } \\ \text { Visual Tracking }\end{array}\end{array}$

Adaptive Decontamination of the Training Set:

Paper F A Unified Formulation for Discriminative Visual 145 Tracking

Paper G Deep Motion and Appearance Cues for Visual 159

$\begin{array}{lll}\text { Paper H } & \begin{array}{l}\text { Beyond Correlation Filters: Learning Continuous } \\ \text { Convolution Operators for Visual Tracking }\end{array} & 171\end{array}$

Paper I ECO: Efficient Convolution Operators for Tracking 195 
PART I

BACKGROUND 



\section{INTRODUCTION}

\subsection{Visual Tracking}

Visual tracking is one of the most fundamental problems in the field of computer vision. It is the task of estimating the trajectory of an object or image region in a sequence of images. Visual tracking has a wide range of important applications, where it often acts as a component in larger computer vision systems. Autonomous driving and vision-based active safety systems rely on tracking the location of vehicles, cyclists and pedestrians. In robotics and autonomous systems, tracking objects of interest is one of the important aspects of visual perception, extracting high-level information from the camera sensor to be used in decision making and navigation.

In addition to robotics related applications, visual tracking is frequently employed in automated video analysis. An example is automatic sports analysis, where the information is first extracted by detecting and tracking the players and objects involved in the game. Other applications include augmented reality and structure-from-motion, where the task is often to track distinctive local image regions. This allows estimating the motion of the camera and constructing a 3D-map of the surrounding world.

As indicated by the variety of applications, the visual tracking problem itself is extremely diverse. Approaches largely depend on the a-priori assumptions, application and sought information. For example, many applications require tracking of certain known object categories, e.g. humans or vehicles. Such a-priori information can be exploited in the design of specialized visual tracking methods intended for a particular application, e.g. tracking of pedestrians. This is often performed by first constructing or learning an appearance model of the object category. In the context of visual tracking this is called offline learning, as the model is constructed prior to its application. 
In a more general form of visual tracking, a-priori information about the object appearance is not known. This is often referred to as generic visual tracking. In contrast to the previous case, a model of the object appearance must be learned online, while the tracking algorithm is applied to the specific video. In this scenario, the tracker is first initialized at an image region that defines the target object. This step is either performed automatically by a detector or by manual supervision. The tracker must then construct or learn an appearance model to be used when searching for the target object in subsequent frames. While generic visual tracking put no or little assumptions on the target appearance, it is often of interest even when the object category is known. An online learned appearance model captures the instance-specific appearance information that is unavailable at the offline learning stage. For instance, an online model learns the specific appearance of an observed human, potentially increasing the performance of a pedestrian tracking system.

Humans are known to excel at visual tracking as we effortlessly perform this vital task in our everyday life. Yet, it has proved remarkably challenging to automate due to several factors. Target objects often change appearance in a complex and non-linear manner that is difficult to model. Environmental factors, such as illumination changes and motion blur distorts the appearance of the target. Moreover, occlusions can cause the target to partially or fully disappear from the view. Lastly, objects or background structures of seemingly similar appearance can be confused with the target itself.

Increasing robustness to the aforementioned factors has been central in visual tracking research, spanning the last few decades. While significant progress have been made, visual tracking is still an open research problem and, perhaps, more active than ever. The last few years have seen a rapid advancement in generic object tracking, in part driven by the introduction of several datasets $[53,54,52,50,51,87,88,60,83,69]$, enabling thorough benchmarking and recording progression over time. The intention of this thesis is to address some of the important problems faced in the pursuit of better robustness and applicability.

\subsection{Contributions}

This thesis primarily studies and advances a class of generic visual tracking methods called Discriminative Correlation Filters (DCF). They were first introduced to the tracking community by Bolme et al. [5], who proposed the MOSSE tracking algorithm in 2010. In essence, DCF methods learn a linear regressor that aims to discriminate the target object from the surrounding background. The key idea is to model the translation invariant application of a linear regressor across an image region as a circular correlation. This allows efficient model inference and prediction by exploiting the Fast Fourier Transform (FFT) algorithm. 
In recent years the DCF-based tracking framework has become incredibly popular, with hundreds of published papers. Its popularity stems from the efficiency, versatility and excellent performance of the DCF framework. This thesis includes several key contributions that have added to its aforementioned advantages. The majority of contributions regard the learning and utilization of the appearance model. These include: (i) Novel model update strategies and optimization techniques to robustly and efficiently infer the appearance model online when using multi-dimensional image features. (ii) A spatial regularization component to reduce the negative effects induced by circular correlation, greatly increasing the discriminative power of the learned model. (iii) A learning formulation that jointly infers the appearance model and the training sample weights, alleviating model drift by reducing the impact of corrupted samples. (iv) A theoretical framework for learning convolution operators in the continuous spatial domain, enabling the integration of multiresolution deep feature maps and sub-pixel localization. (v) Dimensionality reduction techniques to reduce computational complexity and overfitting. (vi) Investigation of the scale estimation problem and the introduction of an efficient one-dimensional scale filter approach.

Another important aspect of DCF tracking investigated in this thesis is the selection and integration of image features. Comprehensive evaluations of color features are performed. Furthermore, deep convolutional features are integrated and investigated for DCF-based tracking. Finally, the impact of deep motion features, computed by applying deep networks to optical flow images, are investigated.

In all included publications, comprehensive experimental analysis and validation is performed using established benchmark datasets and protocols. Several proposed trackers set new state-of-the-art results and have achieved top ranks in independent challenges and evaluations. The DSST tracker, proposed in [13] and Paper C, won the Visual Object Tracking (VOT) challenge 2014 [54]. The SRDCF tracker (Paper D) achieved top rank in the VOT Thermal Infrared challenge 2015 [26] and won the OpenCV State of the Art Vision Challenge [72]. Lastly, the C-COT introduced in Paper H achieved the top rank in VOT2016 [50] and the sequestered dataset of VOT2017 [51].

\subsection{Outline}

This thesis is organized into two parts. Part I contains five chapters, intended as a background and overview of the contributions. Chapter 2 includes an introduction to visual tracking, presents underlying theory and introduces the MOSSE tracker. Chapter 3 contains an overview of the DCF framework and introduces the main contributions of this thesis. An overview of employed image features is given in chapter 4. Finally, concluding remarks are stated in chapter 5. Part II contains the publications included in this thesis. 


\subsection{Included Publications}

\section{Paper A: Adaptive Color Attributes for Real-Time Visual Tracking}

Martin Danelljan, Fahad Shahbaz Khan, Michael Felsberg, and Joost van de Weijer. "Adaptive Color Attributes for Real-Time Visual Tracking". In: IEEE Conference on Computer Vision and Pattern Recognition, CVPR 2014, Columbus, OH, USA, June 23-28. 2014, pp. 1090-1097. DOI: 10 . 1109/CVPR.2014.143 Accepted as oral presentation (6\% acceptance rate).
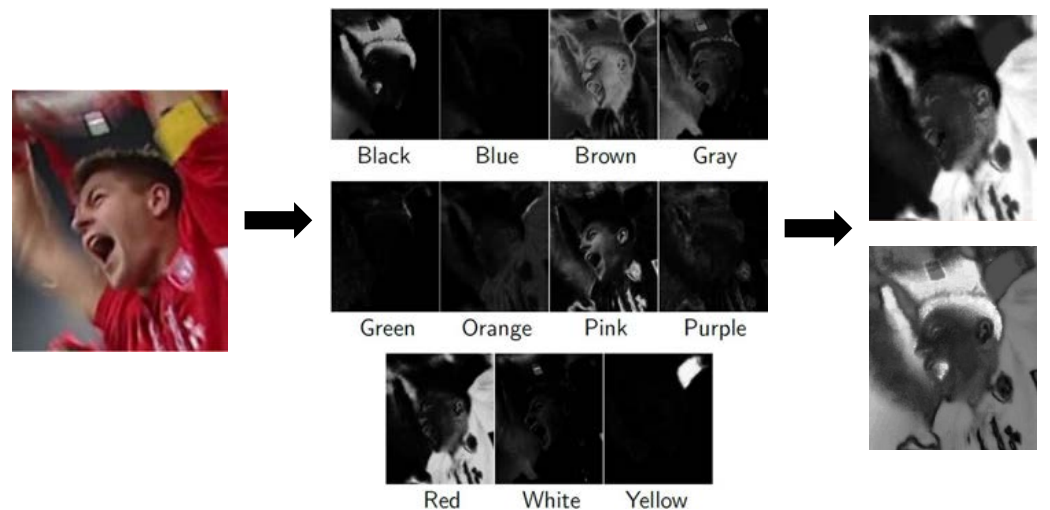

Abstract: Visual tracking is a challenging problem in computer vision. Most state-of-the-art visual trackers either rely on luminance information or use simple color representations for image description. Contrary to visual tracking, for object recognition and detection, sophisticated color features when combined with luminance have shown to provide excellent performance. Due to the complexity of the tracking problem, the desired color feature should be computationally efficient, and possess a certain amount of photometric invariance while maintaining high discriminative power.

This paper investigates the contribution of color in a tracking-by-detection framework. Our results suggest that color attributes provides superior performance for visual tracking. We further propose an adaptive lowdimensional variant of color attributes. Both quantitative and attribute-based evaluations are performed on 41 challenging benchmark color sequences. The proposed approach improves the baseline intensity-based tracker by $24 \%$ in median distance precision. Furthermore, we show that our approach outperforms state-of-the-art tracking methods while running at more than 100 frames per second.

Author's contributions: The author developed the methods, conducted the experiments and was the main contributor to the manuscript. The initial idea was developed by the author together with Fahad Khan. 


\section{Paper B: Coloring Channel Representations for Visual Tracking}

Martin Danelljan, Gustav Häger, Fahad Shahbaz Khan, and Michael Felsberg. "Coloring Channel Representations for Visual Tracking". In: 19th Scandinavian Conference on Image Analysis, SCIA 2015, Copenhagen, Denmark, June 15-17. 2015, pp. 117-129. DOI: $10.1007 / 978-3-319-$ 19665-7_10

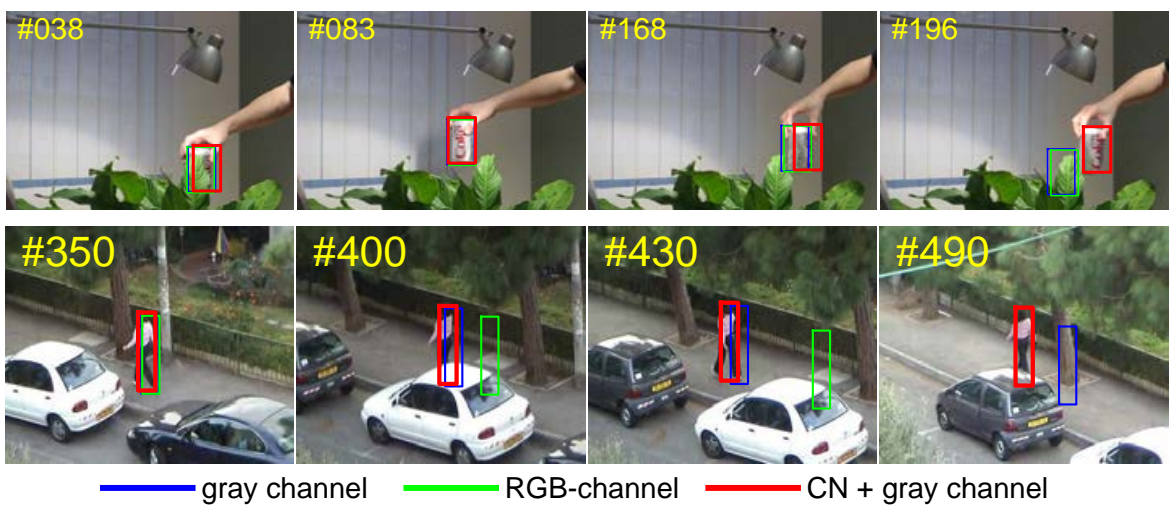

Abstract: Visual object tracking is a classical, but still open research problem in computer vision, with many real world applications. The problem is challenging due to several factors, such as illumination variation, occlusions, camera motion and appearance changes. Such problems can be alleviated by constructing robust, discriminative and computationally efficient visual features. Recently, biologically-inspired channel representations [27] have shown to provide promising results in many applications ranging from autonomous driving to visual tracking.

This paper investigates the problem of coloring channel representations for visual tracking. We evaluate two strategies, channel concatenation and channel product, to construct channel coded color representations. The proposed channel coded color representations are generic and can be used beyond tracking.

Experiments are performed on 41 challenging benchmark videos. Our experiments clearly suggest that a careful selection of color feature together with an optimal fusion strategy, significantly outperforms the standard luminance based channel representation. Finally, we show promising results compared to state-of-the-art tracking methods in the literature.

Author's contributions: The author was the main contributor to the manuscript and the initial idea. Implementation and experiments were performed by the author in collaboration with Gustav Häger. 


\section{Paper C: Discriminative Scale Space Tracking}

Martin Danelljan, Gustav Häger, Fahad Shahbaz Khan, and Michael Felsberg. "Discriminative Scale Space Tracking". In: IEEE Transactions on Pattern Analysis and Machine Intelligence 39.8 (2017), pp. 1561-1575. DOI: $10.1109 /$ TPAMI .2016 .2609928

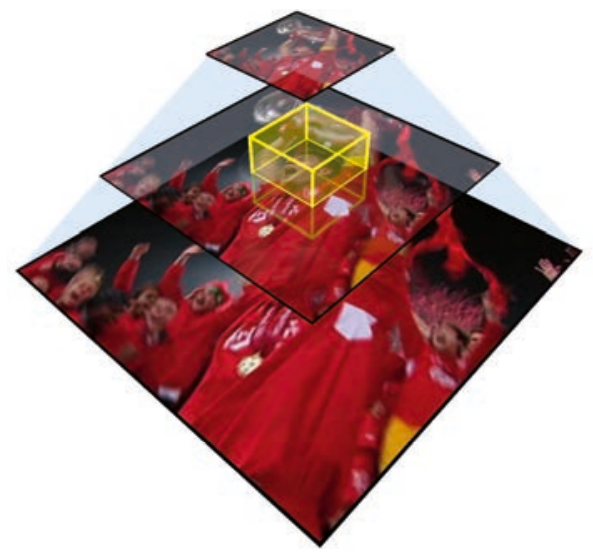

Abstract: Accurate scale estimation of a target is a challenging research problem in visual object tracking. Most state-of-the-art methods employ an exhaustive scale search to estimate the target size. The exhaustive search strategy is computationally expensive and struggles when encountered with large scale variations. This paper investigates the problem of accurate and robust scale estimation in a tracking-by-detection framework. We propose a novel scale adaptive tracking approach by learning separate discriminative correlation filters for translation and scale estimation. The explicit scale filter is learned online using the target appearance sampled at a set of different scales. Contrary to standard approaches, our method directly learns the appearance change induced by variations in the target scale. Additionally, we investigate strategies to reduce the computational cost of our approach.

Extensive experiments are performed on the OTB and the VOT2014 datasets. Compared to the standard exhaustive scale search, our approach achieves a gain of $2.5 \%$ in average overlap precision on the OTB dataset. Additionally, our method is computationally efficient, operating at a $50 \%$ higher frame rate compared to the exhaustive scale search. Our method obtains the top rank in performance by outperforming 19 state-of-the-art trackers on OTB and 37 state-of-the-art trackers on VOT2014.

Author's contributions: The author was the main contributor to the manuscript and the initial idea. Implementation and experiments were performed by the author in collaboration with Gustav Häger. 


\title{
Paper D: Learning Spatially Regularized Correlation Filters for Visual Tracking
}

Martin Danelljan, Gustav Häger, Fahad Shahbaz Khan, and Michael Felsberg. "Learning Spatially Regularized Correlation Filters for Visual Tracking". In: IEEE International Conference on Computer Vision, ICCV 2015, Santiago, Chile, December 7-13. 2015, pp. 4310-4318. DOI: 10.1109/ ICCV. 2015.490

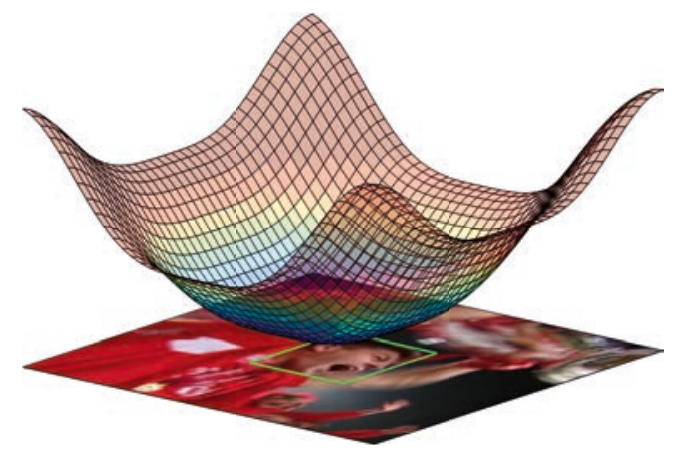

\begin{abstract}
Robust and accurate visual tracking is one of the most challenging computer vision problems. Due to the inherent lack of training data, a robust approach for constructing a target appearance model is crucial. Recently, discriminatively learned correlation filters (DCF) have been successfully applied to address this problem for tracking. These methods utilize a periodic assumption of the training samples to efficiently learn a classifier on all patches in the target neighborhood. However, the periodic assumption also introduces unwanted boundary effects, which severely degrade the quality of the tracking model.

We propose Spatially Regularized Discriminative Correlation Filters (SRDCF) for tracking. A spatial regularization component is introduced in the learning to penalize correlation filter coefficients depending on their spatial location. Our SRDCF formulation allows the correlation filters to be learned on a significantly larger set of negative training samples, without corrupting the positive samples. We further propose an optimization strategy, based on the iterative Gauss-Seidel method, for efficient online learning of our SRDCF. Experiments are performed on four benchmark datasets: OTB-2013, ALOV++, OTB-2015, and VOT2014. Our approach achieves state-of-theart results on all four datasets. On OTB-2013 and OTB-2015, we obtain an absolute gain of $8.0 \%$ and $8.2 \%$ respectively, in mean overlap precision, compared to the best existing trackers.
\end{abstract}

Author's contributions: The author initiated and developed the method, conducted the experiments and was the main contributor to the manuscript and implementation. 


\title{
Paper E: Convolutional Features for Correlation Filter Based Visual Tracking
}

Martin Danelljan, Gustav Häger, Fahad Shahbaz Khan, and Michael Felsberg. "Convolutional Features for Correlation Filter Based Visual Tracking". In: IEEE International Conference on Computer Vision ICCV Workshop 2015, Santiago, Chile, December 7-13. 2015, pp. 621-629. DOI: 10.1109/ICCVW. 2015.84

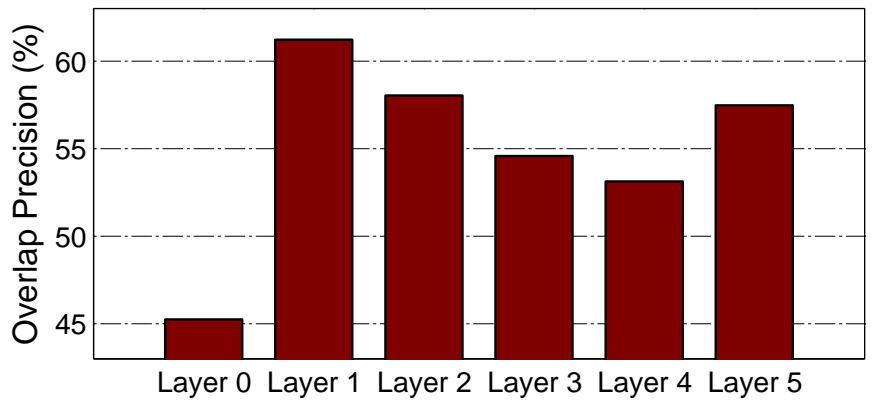

\begin{abstract}
Visual object tracking is a challenging computer vision problem with numerous real-world applications. This paper investigates the impact of convolutional features for the visual tracking problem. We propose to use activations from the convolutional layer of a CNN in discriminative correlation filter based tracking frameworks. These activations have several advantages compared to the standard deep features (fully connected layers). Firstly, they mitigate the need of task specific fine-tuning. Secondly, they contain structural information crucial for the tracking problem. Lastly, these activations have low dimensionality.

We perform comprehensive experiments on three benchmark datasets: OTB, ALOV300++ and the recently introduced VOT2015. Surprisingly, different to image classification, our results suggest that activations from the first layer provide superior tracking performance compared to the deeper layers. Our results further show that the convolutional features provide improved results compared to standard hand-crafted features. Finally, results comparable to state-of-the-art trackers are obtained on all three benchmark datasets.
\end{abstract}

Author's contributions: The author and Gustav Häger equally contributed to the manuscript, idea, implementation and experiments. 


\section{Paper F: Adaptive Decontamination of the Training Set: A Unified Formulation for Discriminative Visual Tracking}

Martin Danelljan, Gustav Häger, Fahad Shahbaz Khan, and Michael Felsberg. "Adaptive Decontamination of the Training Set: A Unified Formulation for Discriminative Visual Tracking". In: IEEE Conference on Computer Vision and Pattern Recognition, CVPR 2016, Las Vegas, NV, USA, June 27-30. 2016, pp. 1430-1438. DOI: 10.1109/CVPR.2016.159
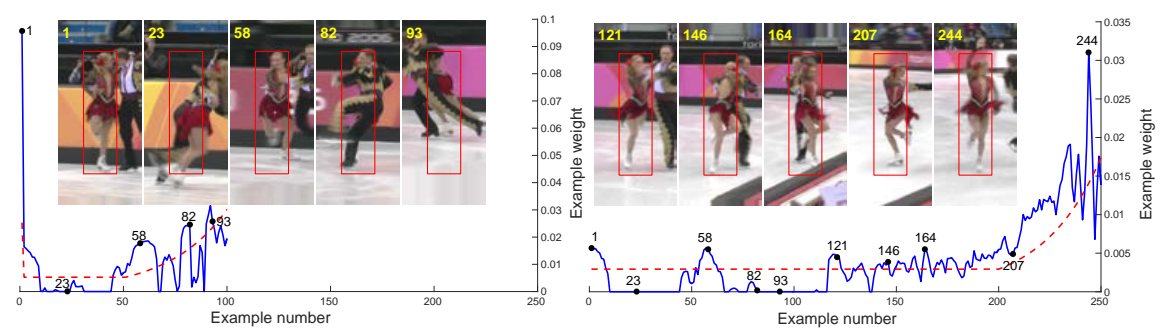

Abstract: Tracking-by-detection methods have demonstrated competitive performance in recent years. In these approaches, the tracking model heavily relies on the quality of the training set. Due to the limited amount of labeled training data, additional samples need to be extracted and labeled by the tracker itself. This often leads to the inclusion of corrupted training samples, due to occlusions, misalignments and other perturbations. Existing trackingby-detection methods either ignore this problem, or employ a separate component for managing the training set.

We propose a novel generic approach for alleviating the problem of corrupted training samples in tracking-by-detection frameworks. Our approach dynamically manages the training set by estimating the quality of the samples. Contrary to existing approaches, we propose a unified formulation by minimizing a single loss over both the target appearance model and the sample quality weights. The joint formulation enables corrupted samples to be down-weighted while increasing the impact of correct ones. Experiments are performed on three benchmarks: OTB-2015 with 100 videos, VOT-2015 with 60 videos, and Temple-Color with 128 videos. On the OTB-2015, our unified formulation significantly improves the baseline, with a gain of $3.8 \%$ in mean overlap precision. Finally, our method achieves state-of-the-art results on all three datasets.

Author's contributions: The author initiated and developed the method, and was the main contributor to the manuscript, implementation and experiments. 


\section{Paper G: Deep Motion and Appearance Cues for Visual Tracking}

Martin Danelljan, Goutam Bhat, Susanna Gladh, Fahad Shahbaz Khan, and Michael Felsberg. "Deep Motion and Appearance Cues for Visual Tracking". In: Pattern Recognition Letters (2018). DOI: $10.1016 / \mathrm{j}$. patrec.2018.03.009 Special issue invited paper as winner of the INTEL Best Scientific Paper Award in the Computer Vision and Robot Vision Track at ICPR 2016.

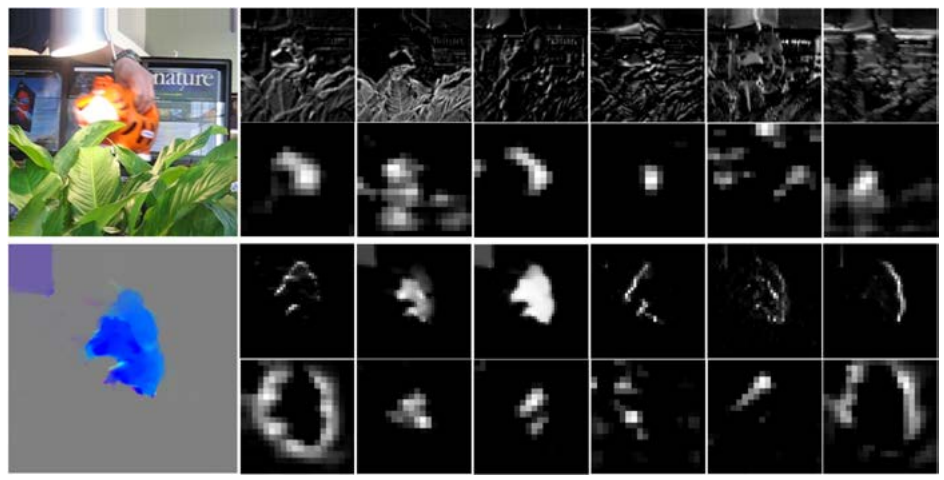

Abstract: Generic visual tracking is a challenging computer vision problem, with numerous applications. Most existing approaches rely on appearance information by employing either hand-crafted features or deep RGB features extracted from convolutional neural networks. Despite their success, these approaches struggle in case of ambiguous appearance information, leading to tracking failure. In such cases, we argue that motion cue provides discriminative and complementary information that can improve tracking performance. Contrary to visual tracking, deep motion features have been successfully applied for action recognition and video classification tasks. Typically, the motion features are learned by training a CNN on optical flow images extracted from large amounts of labeled videos. In this paper, we investigate the impact of deep motion features in a tracking-by-detection framework. We also evaluate the fusion of hand-crafted, deep RGB, and deep motion features and show that they contain complementary information. To the best of our knowledge, we are the first to propose fusing appearance information with deep motion features for visual tracking. Comprehensive experiments clearly demonstrate that our fusion approach with deep motion features outperforms standard methods relying on appearance information alone.

Author's contributions: The author was the main contributor to the manuscript. Implementation and experiments were performed by the author in collaboration with Susanna Gladh and Goutam Bhat. The idea originated from Fahad Khan 


\title{
Paper H: Beyond Correlation Filters: Learning Continuous Convolution Operators for Visual Tracking
}

Martin Danelljan, Andreas Robinson, Fahad Shahbaz Khan, and Michael Felsberg. "Beyond Correlation Filters: Learning Continuous Convolution Operators for Visual Tracking". In: 14th European Conference on Computer Vision ECCV, Amsterdam, The Netherlands, October 11-14, 2016, Proceedings, Part V. 2016, pp. 472-488. DOI: 10.1007/978-3-31946454-1_29 Accepted as oral presentation (2\% acceptance rate).

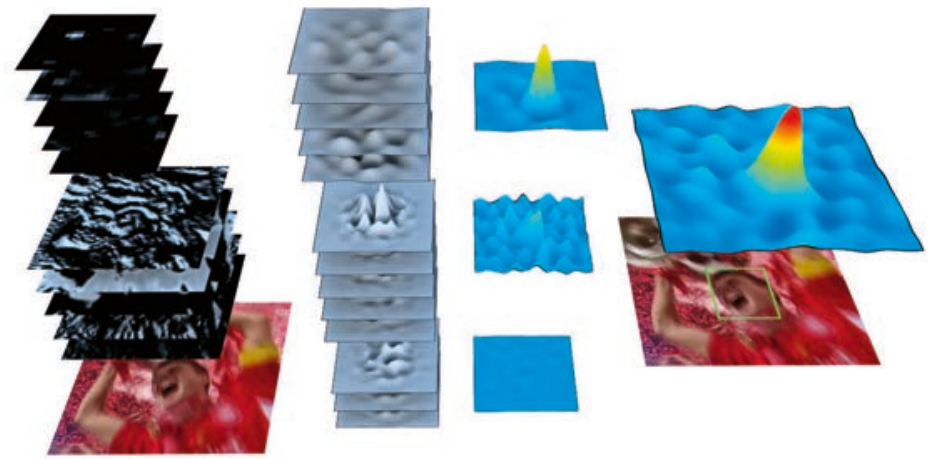

\begin{abstract}
Discriminative Correlation Filters (DCF) have demonstrated excellent performance for visual object tracking. The key to their success is the ability to efficiently exploit available negative data by including all shifted versions of a training sample. However, the underlying DCF formulation is restricted to single-resolution feature maps, significantly limiting its potential. In this paper, we go beyond the conventional DCF framework and introduce a novel formulation for training continuous convolution filters. We employ an implicit interpolation model to pose the learning problem in the continuous spatial domain. Our proposed formulation enables efficient integration of multi-resolution deep feature maps, leading to superior results on three object tracking benchmarks: OTB-2015 (+5.1\% in mean OP), Temple-Color $(+4.6 \%$ in mean OP), and VOT2015 (20\% relative reduction in failure rate). Additionally, our approach is capable of sub-pixel localization, crucial for the task of accurate feature point tracking. We also demonstrate the effectiveness of our learning formulation in extensive feature point tracking experiments.
\end{abstract}

Author's contributions: The author initiated and developed the method, conducted the experiments and was the main contributor to the manuscript. The implementation of the feature point tracker was performed in collaboration with Andreas Robinson. 


\section{Paper I: ECO: Efficient Convolution Operators for Tracking}

Martin Danelljan, Goutam Bhat, Fahad Shahbaz Khan, and Michael Felsberg. "ECO: Efficient Convolution Operators for Tracking". In: IEEE Conference on Computer Vision and Pattern Recognition CVPR 2017, Honolulu, HI, USA, July 21-26. 2017, pp. 6931-6939. DOI: 10.1109/ CVPR. 2017.733
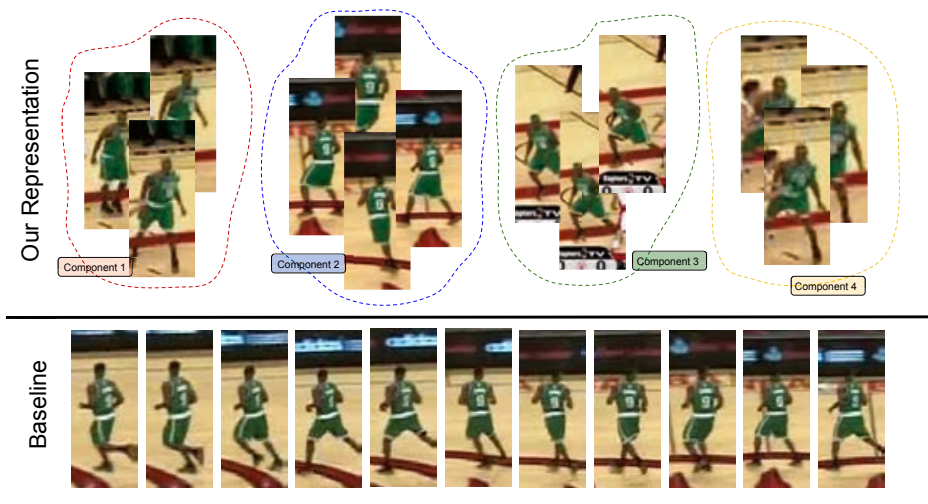

Abstract: In recent years, Discriminative Correlation Filter (DCF) based methods have significantly advanced the state-of-the-art in tracking. However, in the pursuit of ever increasing tracking performance, their characteristic speed and real-time capability have gradually faded. Further, the increasingly complex models, with massive number of trainable parameters, have introduced the risk of severe over-fitting. In this work, we tackle the key causes behind the problems of computational complexity and over-fitting, with the aim of simultaneously improving both speed and performance.

We revisit the core DCF formulation and introduce: (i) a factorized convolution operator, which drastically reduces the number of parameters in the model; (ii) a compact generative model of the training sample distribution, that significantly reduces memory and time complexity, while providing better diversity of samples; (iii) a conservative model update strategy with improved robustness and reduced complexity. We perform comprehensive experiments on four benchmarks: VOT2016, UAV123, OTB-2015, and TempleColor. When using expensive deep features, our tracker provides a 20-fold speedup and achieves a 13.0\% relative gain in Expected Average Overlap compared to the top ranked method [23] in the VOT2016 challenge. Moreover, our fast variant, using hand-crafted features, operates at $60 \mathrm{~Hz}$ on a single CPU, while obtaining $65.0 \%$ AUC on OTB-2015.

Author's contributions: The author developed the method and was the main contributor to the manuscript. Implementation and experiments were performed by the author in collaboration with Goutam Bhat. 


\subsection{Additional Publications}

This section lists peer-reviewed publications by the author that are not included in this thesis. The list is organized into three parts.

\section{Preliminary versions of included publications:}

Two included publications are journal extensions of conference papers.

Martin Danelljan, Gustav Häger, Fahad Shahbaz Khan, and Michael Felsberg. "Accurate Scale Estimation for Robust Visual Tracking". In: British Machine Vision Conference, BMVC 2014, Nottingham, UK, September 1-5. 2014. URL: http://www. bmva.org/bmvc/2014/papers/ paper038/index.html

Paper $\mathrm{C}$ is the journal extension of the above paper. Compared to this preliminary version, the manuscript in Paper $\mathrm{C}$ was rewritten and substantially extended. It includes a thorough investigation of different scale estimation techniques in DCF, additional novelty, more detailed theoretical background and more extensive experimental analysis and validation.

Susanna Gladh, Martin Danelljan, Fahad Shahbaz Khan, and Michael Felsberg. "Deep motion features for visual tracking". In: 23rd International Conference on Pattern Recognition, ICPR 2016, Cancún, Mexico, December 4-8. 2016, pp. 1243-1248. DOI: 10.1109/ICPR. 2016.7899807 Best paper award in the Computer Vision and Robot Vision track.

Paper $\mathrm{G}$ is the journal extension of the above paper. It was primarily extended with more detailed analysis and experimental validation.

\section{Additional related publications:}

The following four publications are related to the subject of this thesis. The first paper propose a deformable DCF model for tracking. The second and third papers apply tracking methods developed in this thesis to Unmanned Aerial Vehicle (UAV) systems. The fourth paper investigates the use of DCF methods for the task of panorama stitching.

Joakim Johnander, Martin Danelljan, Fahad Shahbaz Khan, and Michael Felsberg. "DCCO: Towards Deformable Continuous Convolution Operators for Visual Tracking". In: 17th International Conference on Computer Analysis of Images and Patterns, CAIP 2017, Ystad, Sweden, August 22-24. 2017, pp. 55-67. DOI: 10.1007/978-3-319-64689-3_5

Martin Danelljan, Fahad Shahbaz Khan, Michael Felsberg, Karl Granström, Fredrik Heintz, Piotr Rudol, Mariusz Wzorek, Jonas Kvarnström, and Patrick Doherty. "A Low-Level Active Vision Framework for Collaborative Unmanned Aircraft Systems". In: European Conference on Computer Vision ECCV Workshops 2016 - Zurich, Switzerland, September 6-7 and 12. 2014, pp. 223-237. DOI: 10.1007/978-3-319-16178-5_15 
Gustav Häger, Goutam Bhat, Martin Danelljan, Fahad Shahbaz Khan, Michael Felsberg, Piotr Rudol, and Patrick Doherty. "Combining Visual Tracking and Person Detection for Long Term Tracking on a UAV". in: 12th International Symposium on Visual Computing, ISVC 2016, Las Vegas, NV, USA, December 12-14. 2016, pp. 557-568. DOI: 10.1007/978-3-319-50835-1_50

Giulia Meneghetti, Martin Danelljan, Michael Felsberg, and Klas Nordberg. "Image Alignment for Panorama Stitching in Sparsely Structured Environments". In: 19th Scandinavian Conference, SCIA 2015, Copenhagen, Denmark, June 15-17. 2015, pp. 428-439. DOI: 10.1007/978-3-319-19665-7_36

\section{Point cloud registration and segmentation:}

The following four publications represent separate lines of research. The first three papers (two CVPR and one ICPR) address the problem of point cloud registration. It is a line of research that has also been driven by the author, but falls outside the scope of this thesis. The first and second paper integrates and investigates color and shape features for probabilistic point cloud registration. The third paper proposes a density adaptive probabilistic model. The fourth paper proposes a framework for deep semantic segmentation of point clouds.

Martin Danelljan, Giulia Meneghetti, Fahad Shahbaz Khan, and Michael Felsberg. "A Probabilistic Framework for Color-Based Point Set Registration". In: IEEE Conference on Computer Vision and Pattern Recognition, CVPR 2016, Las Vegas, NV, USA, June 27-30. 2016, pp. 1818-1826. DOI: 10.1109/CVPR.2016.201

Martin Danelljan, Giulia Meneghetti, Fahad Shahbaz Khan, and Michael Felsberg. "Aligning the dissimilar: A probabilistic method for featurebased point set registration". In: 23rd International Conference on Pattern Recognition, ICPR 2016, Cancún, Mexico, December 4-8. 2016, pp. 247-252. DOI: 10.1109/ICPR.2016.7899641

Felix Järemo Lawin, Martin Danelljan, Fahad Shahbaz Khan, Per-Erik Forssén, and Michael Felsberg. "A Probabilistic Framework for ColorBased Point Set Registration". In: IEEE Conference on Computer Vision and Pattern Recognition, CVPR 2018, Salt Lake City, UT, USA, June 18-22. 2018 Accepted as oral presentation.

Felix Järemo Lawin, Martin Danelljan, Patrik Tosteberg, Goutam Bhat, Fahad Shahbaz Khan, and Michael Felsberg. "Deep Projective 3D Semantic Segmentation". In: 17th International Conference on Computer Analysis of Images and Patterns, CAIP 2017, Ystad, Sweden, August 22-24. 2017, pp. 95-107. DOI: 10.1007/978-3-319-64689-3_8 


\section{From LuCAS-KANADE TO MOSSE}

This chapter is intended as a background to Discriminative Correlation Filters (DCF) and the contributions of this thesis. When developing the underlying theory of DCF, one strategy is to focus on the correlation filter part of the story by revisiting countless correlation filter variants $[55,46,24,6,43,56$, $57,66,67,78]$. While these works were important steps in the development of the MOSSE tracker [5], they are not central in the understanding of DCF trackers in general, and how they differ from other visual tracking paradigms. In this chapter, I therefore choose to focus on the discriminative part of the story by first relating DCF to the most classical, yet still applied tracking and image registration techniques: the Lucas-Kanade (LK) algorithm [63] and Normalized Cross Correlation (NCC). To explore the fundamental differences and developments, the focus of this chapter is on the underlying tracking models and not the algorithmic details.

\subsection{A Generative Tracking Model}

For this presentation, we will take the probabilistic perspective to clarify the underlying assumptions embedded in the revisited tracking approaches. This perspective also serves the purpose of highlighting the key difference between generative and discriminative models for tracking. We will start by considering an image containing a region of interest, as depicted in figure 2.1. The aim could be to locate this region in an other image or a consecutive sequence of images. In the former case one would refer to the task as image registration, while the latter task is generally called tracking. The region of interest could, for example, constitute a specific target object, an interest point, or the entire image itself. In any case, this region is referred to as the target patch, or simply the target.

Let us denote the extracted target patch by $x$. We consider $x$ to be a grayscale image patch for simplicity. For convenience, let this be a rectangular 

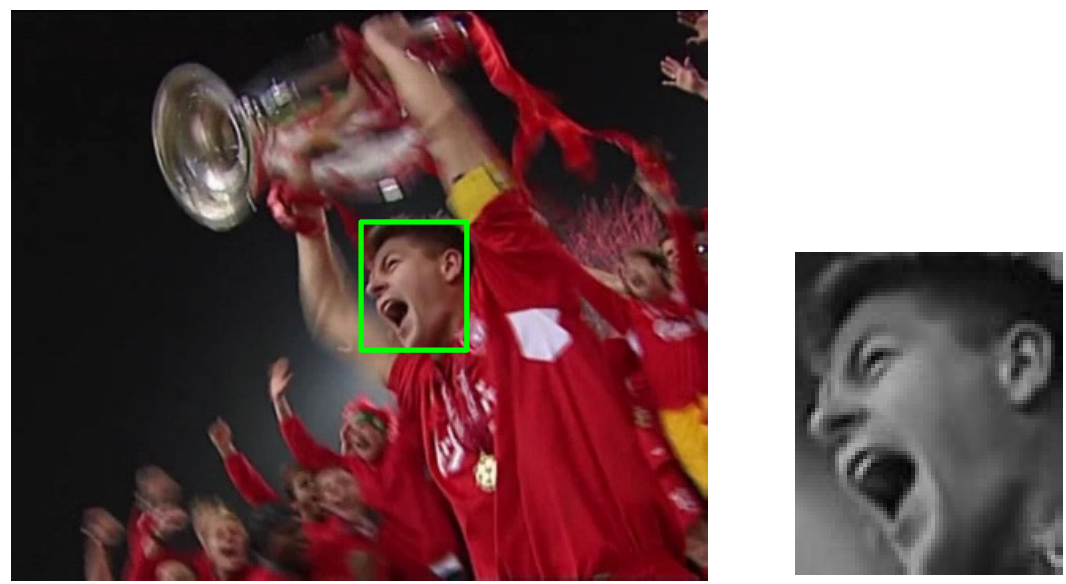

Figure 2.1: The original image (left) containing the target region of interest (green box). The target grayscale patch $x$ (right) is extracted from this region.

region of the image with a width and height of $N_{1}$ and $N_{2}$ pixels respectively. Mostly, we think of $x$ as a function $x:\left\{1, \ldots, N_{1}\right\} \times\left\{1, \ldots, N_{2}\right\} \rightarrow \mathbb{R}$, where $x\left(n_{1}, n_{2}\right)=x(n) \in \mathbb{R}$ is the intensity value of the pixel at coordinate $n=$ $\left(n_{1}, n_{2}\right)$. Sometimes it is more suitable to think of $x$ as an element of $\mathbb{R}^{N}$, i.e. as a vectorization of the pixel values, where $N=N_{1} N_{2}$ is the total number of pixels within the patch. To avoid cluttering the notation, we will let the interpretation of $x$ be clear from the context when important. Of course, there exist a trivial isomorphism between these two representations.

As our task is to locate the patch $x$ in another image, we first construct an appearance model of the target. As a second step, we employ the appearance model in the search for the corresponding region in the other image. As a first choice, we model the joint probability density function $p(x)$ of the pixel values in the region of interest. Such models are called generative, as they describe the observed data $x$. In principle, we can use the generative model $p(x)$ to generate different versions of the target patch $x$ by sampling from the distribution $p(x)$.

There, of course, exist a vast number of alternative generative models for tracking, not all of which explicitly model $p(x)$. Here, we will first consider one of the simplest. We let $p(x)$ be modeled by a multivariate Gaussian distribution $p(x)=\mathcal{N}\left(x ; \mu, \sigma^{2} I\right)$ with an expectation $\mu \in \mathbb{R}^{N}$ and covariance $\sigma^{2} I$, where $I$ denotes the identity matrix. For simplicity, we take the variance $\sigma^{2}$ as a scalar hyper-parameter. The mean parameter $\mu$ can be inferred by Maximum Likelihood,

$$
\underset{\mu}{\arg \max } p(x \mid \mu)=\underset{\mu}{\arg \min }-\log p(x \mid \mu) .
$$


Given only a single observation $x$ of the target patch, we easily find the solution to be $\mu=x$.

After the appearance model is constructed, we set out to locate the target patch in a new image $J$. As for $x$, we see $J$ as a function $J\left(t_{1}, t_{2}\right)$ of two spatial variables. But in this case, they range over all pixels in the image. We further let $\left(t_{1}, t_{2}\right) \in \mathbb{R}^{2}$, using interpolation to define the intensity value of $J$ at subpixel locations. We restrict our aim to finding the relative translation $u \in \mathbb{R}^{2}$ between the target patch $x$ and the corresponding region in image $J$. Let $z_{u}$ be the $N_{1} \times N_{2}$ image patch extracted at location $u$ in $J$, that is $z_{u}(n)=J(n+u)$. We can estimate the translation $u$ by finding the patch $z_{u}$ that best fits our generative appearance model $p(x \mid \mu)$,

$$
\underset{u}{\arg \max } p\left(z_{u} \mid \mu\right)
$$

By taking the negative logarithm of (2.2) and ignoring constant terms and factors, we can equivalently minimize the loss function,

$$
L(u)=\left\|z_{u}-\mu\right\|^{2}=\sum_{n}(J(n+u)-\mu(n))^{2} .
$$

We thereby arrive in the familiar squared error, also known as the $L^{2}$ metric. The simplest method of minimizing the loss (2.3) is to perform a grid search over the translation $u$. In this context, such a strategy is known as block-matching, exhibiting an infamous squared complexity in the region size $N$. To tackle this problem, Lucas and Kanade [63] came up with the ingenuous idea of applying the Gauss-Newton algorithm to the loss above,

$$
L(u) \approx \sum_{n}\left(J\left(n+u_{0}\right)+\nabla J\left(n+u_{0}\right)^{\mathrm{T}} h-\mu(n)\right)^{2} .
$$

Here, $\nabla J$ denotes the gradient of the image. In the equation (2.4) above, the current estimate $u_{0}$ of the translation serves as the point of linearization of the residuals. The approximate loss is now a linear least squares problem in the translation increment $h=u-u_{0}$, which is solved as $h=A^{-1} b$ where,

$$
A=\sum_{n} \nabla J\left(n+u_{0}\right) \nabla J\left(n+u_{0}\right)^{\mathrm{T}}, \quad b=\sum_{n} \nabla J\left(n+u_{0}\right)\left(\mu(n)-J\left(n+u_{0}\right)\right) .
$$

We then update the estimated translation as $u_{0} \leftarrow u_{0}+h$ and iterate the process.

To increase the robustness of the loss (2.3) to illumination and exposure variations, we may consider first normalizing the images based on the local image intensity. That is, we compute the normalized target patch $\tilde{x}=x /\|x\|$, and similarly normalize the candidate patch $\tilde{z}_{u}=z_{u} /\left\|z_{u}\right\|$ at location $u$ in image $J$. In this case, our generative model $p(\tilde{x} \mid \tilde{\mu})$ of the intensity-normalized target appearance takes the mean $\tilde{\mu}=\tilde{x}$, and the corresponding loss (2.3) 
becomes,

$$
L(u)=\left\|\tilde{z}_{u}-\tilde{\mu}\right\|^{2}=2-2\left\langle\tilde{z}_{u}, \tilde{\mu}\right\rangle=2-2 \sum_{n} \frac{J(n+u) x(n)}{\sqrt{\sum_{k} J(k+u)^{2}} \sqrt{\sum_{k} x(k)^{2}}} .
$$

Here, $\langle\cdot, \cdot\rangle$ denotes the standard inner product in $\mathbb{R}^{N}$. We see that $(2.6)$ is minimized by equivalently maximizing the normalized cross-correlation (NCC), defined by the second term after the last equality, between the target patch $x$ and the image $J$. Hence, our simple generative model $p(x)$ gives rise to two of the most well known techniques in computer vision and image analysis: Lucas-Kanade and NCC.

\subsection{Incorporating Background Information}

Now we have seen the close relationship between the generative models of the target appearance employed in the LK and NCC trackers. We conclude that their underlying models are essentially the same, and that the methods only differ in the optimization algorithm applied for finding the translation $u$ in (2.2). While these algorithms have been, and still are widely used in computer vision and image analysis, they are often too simplistic for object tracking. One major limitation is that the appearance model $p(x)$ is completely agnostic to the image background. In this case, the image background refers to the parts of the image that falls outside the region of interest (see figure 2.1). It is not uncommon that the background contains regions or objects, that are similar to the target. Such regions are often called distractors, since they are easily confused with the target itself. By ignoring distractors in the construction of our appearance model, we also neglect the important details that distinguish our target from the appearance of a distractor. Ideally, these details should be taken into account, or even emphasized by the model.

Before moving on, we note that the generative model $p(x \mid \mu)$ described here can be learned from a set $\left\{x_{j}\right\}_{j=1}^{m}$ of samples $x_{j}$ of the target. In this case, we would simply obtain the ML estimate $\mu=\frac{1}{m} \sum_{j} x_{j}$. In practice, the samples $x_{j}$ could be collected from the same image by applying different data augmentation techniques, such as small rotations, to the target patch. An alternative is to collect the samples $x_{j}$ from different images, where the target location is known or estimated.

Building on top of the previous model, the idea of additionally modeling the statistics of the background comes to mind. We can, of course, collect image patches from the background, in addition to the target itself. Let the binary variable $y_{j}$ indicate if the image patch $x_{j}$ represent the target or background by taking the value $y_{j}=1$ and $y_{j}=0$ respectively (see figure 2.2). We will consider these two as different classes. Our new dataset $\mathcal{X}=\left\{\left(x_{j}, y_{j}\right)\right\}_{j=1}^{m}$ contains observations of both the target and background appearance. These are assumed to be stochastically independent to simplify 


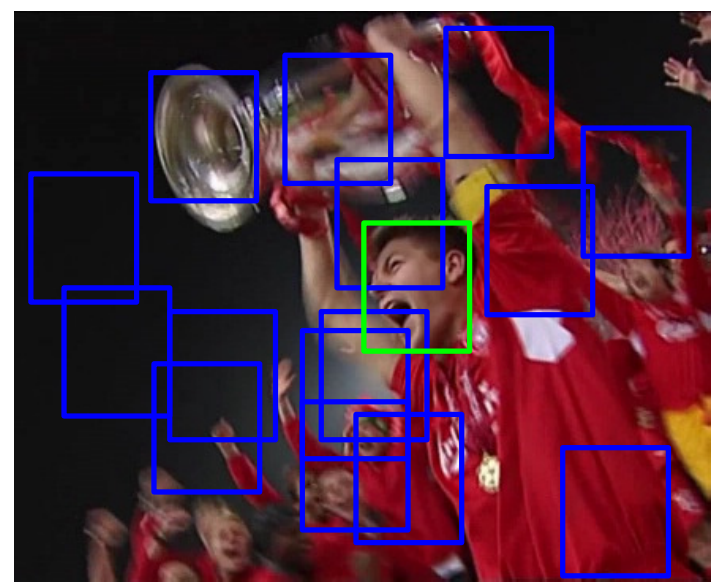

Figure 2.2: Sample image patches $x_{j}$ of both the target (green box) and background (blue boxes). The target patch is given the target value $y_{j}=1$, while the background patches are set to $y_{j}=0$.

inference. Our model is extended to describe the joint probability density $p(x, y)$. By utilizing the factorization $p(x, y)=p(x \mid y) p(y)$, each factor can be modeled separately. For simplicity, consider a uniform prior probability of the classes $p(y=0)=p(y=1)=\frac{1}{2}$. As previously, the class-conditional distributions of the image patch $x$ are modeled as a multi-variate Gaussian $p(x \mid y=c, \theta)=\mathcal{N}\left(x ; \mu_{c}, \sigma^{2} I\right)$ for $c \in\{0,1\}$. Here, we use $\theta=\left(\mu_{0}, \mu_{1}\right)$ as a compact notation for all the parameters in the model.

We can infer the parameters of our new model $p(x, y \mid \theta)$ by extending the ML methodology applied in (2.1) and taking the negative logarithm,

$$
\underset{\theta}{\arg \max } \prod_{j=1}^{m} p\left(x_{j}, y_{j} \mid \theta\right)=\underset{\theta}{\arg \min } \sum_{c=0}^{1} \sum_{j: y_{j}=c}\left\|x_{j}-\mu_{c}\right\|^{2} .
$$

We directly obtain $\mu_{c}=\frac{1}{m_{c}} \sum_{j: y_{j}=c} x_{j}$ as the empirical mean of all $m_{c}$ samples from class $c$. With our new appearance model constructed, we can classify a candidate patch $z_{u}$ by evaluating the probability of it being the target. This is performed by a simple application of Bayes formula,

$$
\begin{aligned}
p\left(y=1 \mid z_{u}, \theta\right) & =\frac{p\left(z_{u} \mid y=1, \theta\right) p(y=1)}{\sum_{c} p\left(z_{u} \mid y=c, \theta\right) p(y=c)} \\
& =\operatorname{sigm}\left(\frac{2\left\langle\mu_{1}-\mu_{0}, z_{u}\right\rangle-\left(\left\|\mu_{1}\right\|^{2}-\left\|\mu_{0}\right\|^{2}\right)}{2 \sigma^{2}}\right) .
\end{aligned}
$$

Here, $\operatorname{sigm}(v)=\frac{1}{1+\exp (-v)}$ denotes the sigmoid function. The second equality is obtained by canceling out and rearranging some factors (see e.g. [70] for a derivation). As desired, the predicted target probability (2.8) takes both 


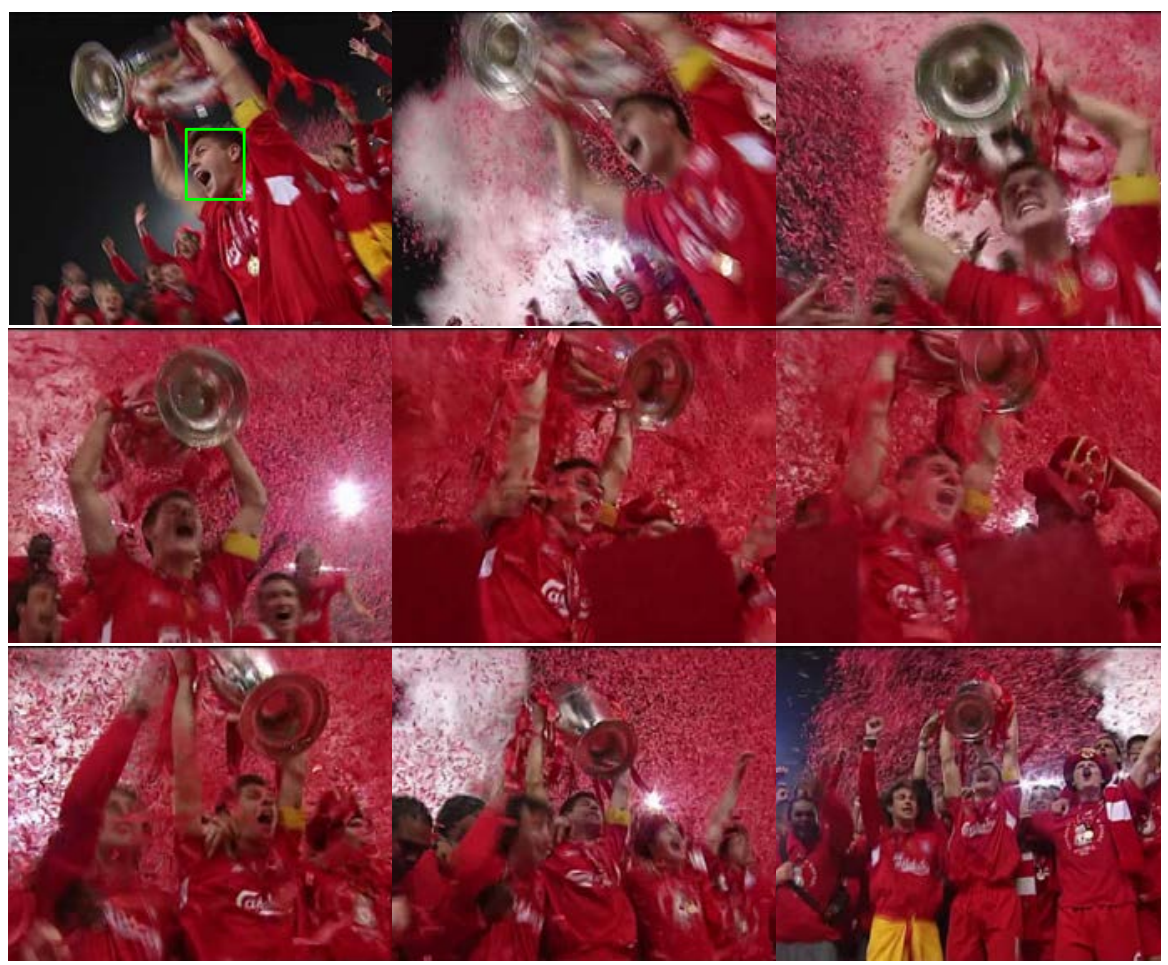

Figure 2.3: The target, marked with a green box in the first frame, undergoes significant appearance changes while affected by motion blur, illumination changes, background clutter and occlusions. The background is even more dynamic.

target and background information into account. By setting $p(y=1 \mid x, \theta)=\frac{1}{2}$, we even find a linear decision boundary between the target and background appearances. In general, this approach is called Linear Discriminant Analysis (LDA) [70], and has seen some applications to visual tracking [61, 30].

\subsection{Generative versus Discriminative Approaches}

Despite its misleading name, LDA is a generative method as it models the full joint distribution $p(x, y)$ of the observed data $x$ and the target variables $y$. Nevertheless, at the localization step (2.8) we only utilize the conditional probability $p(y \mid x)$ of the class variable $y$ given the observed image patch $x$. It seems as we have taken a slight detour by first modeling the appearance of the target $p(x \mid y=1)$ and background $p(x \mid y=0)$. And then deriving a decision criterion (2.8) which is used in tracking. But what is wrong with that? The problem is the assumptions we make in finding tractable generative models. 
In this case, our model is based on the key assumption that the target and background patches $x$ are normally distributed. While this model is ideal if the image is static and only corrupted by additive Gaussian noise, it rarely captures the complexity of the real world. Of course, one can employ more sophisticated generative models, using for instance sparse representations [62, 1], subspace methods [79] or mixtures of fix basis functions [81]. But the main problem still remains: image data is notoriously hard to model. Consider for example coming up with a generative model of the target appearance in figure 2.3. The observed image patch of the target (the face of Steven Gerrard) is affected by motion blur, compression artifacts, partial occlusions, out-of-plane rotations, different illumination conditions. The reader should understand that it is extremely difficult to find a suitable noise model or family of probability distributions that can capture all these variations. To make the task even more challenging, the proposed model should be applicable to any kind of target object and it must be inferred given minimal amounts of data, even only a single image. Furthermore, the approach should model the background appearance, which is often even more diverse and dynamic than the target itself.

Considering all the difficulties of finding a suitable model of the observed image data $x$, it is tempting to model the conditional distribution $p(y \mid x)$ of the target variable $y$ given the image patch $x$ directly. Such models are called discriminative. The main advantage of discriminative models is that they are solving an easier problem, not requiring $p(x)$. However, it is not in my intention to claim that discriminative models are superior in all aspects and applications. The model of the observed data $p(x \mid y)$ is very useful in some applications. Even in tracking, it can be used to study the learned appearance by for instance sampling from the inferred distribution. This is unfortunately not possible in discriminative models. Generative models can more easily handle missing data at the inference stage, while discriminative models mostly can be trained to be robust to it. This is important in case of partial occlusions in tracking. On the other hand, discriminative models are often agnostic to the preprocessing of the observed data. This mean that we can easily integrate powerful feature representations. Instead, modifying the input data in generative models may require changing or even rethinking the model itself as the distribution of the observations have changed.

\subsection{A Discriminative Tracking Model}

Inspired by the LDA-based model in (2.8), the first discriminative model that comes to mind can be constructed as follows. We assume the same dataset $\mathcal{X}=\left\{\left(x_{j}, y_{j}\right)\right\}_{1}^{m}$ as in the previous case. The vector $\sigma^{-2}\left(\mu_{1}-\mu_{0}\right)$ in $(2.8)$ is replaced by a vector $f$ of trainable parameters. Similarly, we replace the bias term $-\frac{1}{2 \sigma^{2}}\left(\left\|\mu_{1}\right\|^{2}-\left\|\mu_{0}\right\|^{2}\right)$ by a trainable scalar $b$. The resulting discriminative 
model is given by $p(y=1 \mid x, \theta)=\operatorname{sigm}(\langle f, x\rangle+b)$, where $\theta=(f, b)$. Using the fact that $p(y=0 \mid x, \theta)=1-p(y=1 \mid x, \theta)$, we infer the parameters $\theta$ by maximizing the likelihood,

$$
\underset{\theta}{\arg \max } \prod_{j=1}^{m} p\left(y_{j} \mid x_{j}, \theta\right)=\underset{\theta}{\arg \min }-\sum_{j=1}^{m} \log p\left(y_{j} \mid x_{j}, \theta\right) .
$$

In contrast to the generative model in (2.7), we here infer the model parameters $\theta$ by maximizing the same decision criterion $p(y \mid x, \theta)$ that we later use for tracking in (2.8). That is, the parameters are learned to discriminate between the target and background appearance on the training set $\mathcal{X}$.

This particular discriminative model is known as Logistic Regression [70]. While popular in general, it is not as common for visual tracking $[74,85]$. Compared to the previously visited methods, the model inference in logistic regression (2.9) does not reduce to a linear least squares problem. It must therefore be tackled with more general optimization techniques, such as Newton or Quasi-Newton methods [71]. Fortunately, it can be shown that the optimization problem (2.9) is convex [70], guaranteeing the existence of a unique optimum and simplifying the convergence.

In contrast to the related computer vision tasks of object detection and recognition, generic visual object tracking is an inherently online task. That is, the model is ultimately inferred online, while the tracker is applied to an image sequence. Furthermore, most tracking applications demand real-time performance. Therefore, we desire the following two properties of a tracking model and its inference algorithm: (1) it should be computationally efficient, (2) it should be easy to update with new data online. The latter means that we can efficiently update the model with new observations without, for instance, having to retrain the model from scratch each time the training set $\mathcal{X}$ is augmented. The logistic regression model certainly satisfies the second property since an iterative optimization method, such as Newtons method, can be "warm started" with the previous estimate of $\theta$. However, inference is not simple and might be computationally demanding.

\subsection{Linear Regression}

To find a model that allows a simpler inference strategy, we first look back at the Lucas-Kanade and LDA methods. We observe that these Gaussian models give rise to pleasant linear least squares ML problems. To achieve this in the previously presented discriminative model, we do another modification. Instead of letting the target variable $y$ be binary, we can let $y \in \mathbb{R}$ be a continuous score value. For instance, a low score of $y \approx 0$ could represent background while a larger score $y \approx 1$ could represent the target. That is, instead of our model predicting the foreground/background class of an image region, we let it predict a target score. The benefit of this modification is that 
we can model the distribution $p(y \mid x)$ of the target score $y$ given an image patch $x$ to be Gaussian, where we in the previous case were forced to use a Bernoulli distribution. Specifically, we let the expectation of $p(y \mid x)$ be a linear function of $x$ and the variance to be controlled by a hyper-parameter $\sigma^{2}$. The model is expressed as,

$$
p(y \mid x, f)=\mathcal{N}\left(y ;\langle f, x\rangle, \sigma^{2}\right)=\frac{1}{\sqrt{2 \pi \sigma^{2}}} \exp \left(-\frac{(y-\langle f, x\rangle)^{2}}{2 \sigma^{2}}\right) .
$$

Here, the vector $f \in \mathbb{R}^{N}$, having the same size $N=N_{1} N_{2}$ as the image patch $x$, contains the only parameters of the model. We can without loss of generality ignore the bias $b$ by extending $x$ with a dummy pixel with a constant value one. Previously, I argued that Gaussianity is a too rigid assumption that does not capture the complexity of the world. But note that in this case, only the target variable $y$ is modeled to be normally distributed while nothing is said about the image data $x$. It is thus a much weaker assumption than the one used in the LDA model.

To infer the parameters of the model (2.10), we again apply the ML methodology,

$$
\underset{f}{\arg \max } \prod_{j=1}^{m} p\left(y_{j} \mid x_{j}, f\right)=\underset{f}{\arg \min }-\sum_{j=1}^{m} \log p\left(y_{j} \mid x_{j}, f\right) .
$$

By inserting (2.10) and discarding unimportant constants, we derive the equivalent loss,

$$
L(f)=\sum_{j=1}^{m}\left(y_{j}-\left\langle f, x_{j}\right\rangle\right)^{2}=\|X f-\mathbf{y}\|^{2} .
$$

Here, $X$ is the $m \times N$ matrix given by $X^{\mathrm{T}}=\left[x_{1}, x_{2}, \cdots, x_{m}\right]$, i.e. the $\mathrm{j}$-th row of $X$ contains the sample $x_{j}$. Similarly, we have defined $\mathbf{y}^{\mathrm{T}}=\left[y_{1}, y_{2}, \cdots, y_{m}\right]$. As in the LDA case (2.7), we are back to a linear least squares problem. In this case, it allows the closed-form solution,

$$
f=\left(X^{\mathrm{T}} X\right)^{-1} X^{\mathrm{T}} \mathbf{y}
$$

Let us assume that the matrix $X^{\mathrm{T}} X$ has full rank, such that the expression above makes sense. We will revisit this issue at a later point.

At the tracking stage, we obtain the Gaussian distribution $p\left(y \mid z_{u}, f\right)=$ $\mathcal{N}\left(y ;\left\langle f, z_{u}\right\rangle, \sigma^{2}\right)$ of the target score at the image patch $z_{u}$. To search for the target in the new image $J$, we thus only need to maximize the expectation of the distribution $p\left(y \mid z_{u}, f\right)$. That is, we want to find the translation $u$ that maximizes the score function,

$$
S(u)=\left\langle f, z_{u}\right\rangle=\sum_{n} J(n+u) f(n)
$$


We note that the tracking can be performed by correlating the image with the filter $f$. Let us compare this result with the generative NCC method in (2.6). While a correlation is performed to locate the target in both cases, the essential difference is that in (2.14) the weights $f$ of the correlation filter has been discriminatively learned to differentiate between the target and background appearances.

\subsection{A Bayesian Perspective on Linear Regression}

Now, let us take a closer look at the solution in (2.13). The first thing to note is that the matrix $X^{\mathrm{T}} X$ is rank deficient if the number of samples $m$ is smaller than the number of pixels $N=N_{1} N_{2}$ in the patch. While this may be of lesser concern in applications where data is of abundance, it a serious issue for generic object tracking. The problem is the limited data available in the first frame of tracking. We often only have a single image to extract patches $x_{j}$ from, limiting the size $m$ of the initial training set. But even if we have harvested the required number of samples $m \geq N$, there may exist linear dependencies in the data. This would in any case lead to a singular or close to singular matrix $X^{\mathrm{T}} X$, resulting in an unstable estimation of $f$.

To counter the lack of data, we integrate a prior probability distribution $p(f)$ of the weights. For our Gaussian likelihood function, the conjugate prior is also a Gaussian, motivating the choice $p(f)=\mathcal{N}\left(f ; 0, \nu^{2} I\right)$. Here, $\nu^{2}$ is a hyper-parameter controlling the prior variance of the filter weights. Inference is performed by maximizing the posterior probability density of the parameters,

$$
p(f \mid \mathcal{X})=\frac{p(\mathbf{y} \mid X, f) p(f)}{p(\mathbf{y} \mid X)} \propto p(\mathbf{y} \mid X, f) p(f)=\left(\prod_{j=1}^{m} p\left(y_{j} \mid x_{j}, f\right)\right) p(f) .
$$

Taking the negative logarithm of the above expression allows us to find the maximum a posteriori (MAP) estimate by minimizing,

$$
L(f)=\|X f-\mathbf{y}\|^{2}+\frac{\sigma^{2}}{\nu^{2}}\|f\|^{2} .
$$

We see that this loss is simply an $L^{2}$-regularized version of (2.12).

With this Bayesian interpretation of the problem, we can actually compute the entire posterior probability density as,

$$
\begin{aligned}
p(f \mid \mathcal{X}) & =\mathcal{N}\left(f ; f_{m}, V_{m}\right) \\
f_{m} & =\left(X^{\mathrm{T}} X+\frac{\sigma^{2}}{\nu^{2}} I\right)^{-1} X^{\mathrm{T}} \mathbf{y} \\
V_{m} & =\left(\frac{1}{\sigma^{2}} X^{\mathrm{T}} X+\frac{1}{\nu^{2}} I\right)^{-1}
\end{aligned}
$$


See e.g. $[70,75]$ for a derivation. Note that the expectation of the posterior (2.17b) coincides with the MAP estimate obtained from (2.16) due to Gaussianity. Finally, we can compute the posterior predictive distribution [70] of a candidate patch $z_{u}$ by integrating out the filter coefficients,

$$
p\left(y \mid z_{u}, \mathcal{X}\right)=\mathcal{N}\left(y ;\left\langle f_{m}, z_{u}\right\rangle, \sigma^{2}+z_{u}^{\mathrm{T}} V_{m} z_{u}\right) .
$$

Note that the expectation of (2.18) coincides with the score function (2.14). We will see that the variance $\sigma^{2}+z_{u}^{\mathrm{T}} V_{m} z_{u}$ of the posterior predictive distribution carries very limited information for our purpose. In the remainder, we will therefore focus on the equations (2.16) and (2.14) in the exploration that follows.

\subsection{Convolution and the Fourier Transform}

We have taken a tour starting from some of the initial visual tracking algorithms: Lucas-Kanade and NCC. Each step has been seemingly small, yet a conceptual leap. We are soon ready for the final step, that will take us to what have served as a starting point for this thesis. But before taking this step, we need to briefly dwell into a subject that is also central to this thesis. Namely, convolutions and its relationship to the Fourier Transform and linear algebra.

Let us consider two real-valued functions $x$ and $f$ defined on a rectangular 2-dimensional grid $\Omega=\left\{0, \ldots, N_{1}-1\right\} \times\left\{0, \ldots, N_{2}-1\right\}$. Note that we here start the indexing with zero for convenience. We first define circular convolution between the functions as,

$$
x * f(n)=\sum_{l_{1}=0}^{N_{1}-1} \sum_{l_{2}=0}^{N_{2}-1} x\left(\left(n_{1}-l_{1}\right)_{N_{1}},\left(n_{2}-l_{2}\right)_{N_{2}}\right) f\left(l_{1}, l_{2}\right), \quad n \in \Omega .
$$

Here, $(a)_{N}=a \bmod N$ is a compact notation for the modulo operation. It is useful to view the convolution (2.19) as an operation $f \mapsto x * f$ applied to $f$, where $x$ is fixed. Since the operation is linear, there exists a matrix $\mathcal{C}(x)$ such that $x * f=\mathcal{C}(x) f$. In the latter expression, both $x * f$ and $f$ are viewed as vectors in $\mathbb{R}^{N}$ using some basis. As mentioned in section 2.1, there is an isomorphism performing this vectorization, by e.g. concatenating the "columns" of the function. However, we will not denote it explicitly to avoid clutter. In the case when $x$ and $f$ are one-dimensional functions, the matrix $\mathcal{C}(x)$ is known as a circulant matrix [45]. In our case, $\mathcal{C}(x)$ will have a circulant block structure, where each block is itself circulant. However, knowledge of the structure of $\mathcal{C}(x)$ is of lesser importance here.

Next, we study the spectral properties of $\mathcal{C}(x)$. For $k=\left(k_{1}, k_{2}\right) \in \Omega$, define the Fourier basis functions as,

$$
e_{k}(n)=e_{k_{1}, k_{2}}\left(n_{1}, n_{2}\right)=\exp \left(i 2 \pi\left(\frac{k_{1} n_{1}}{N_{1}}+\frac{k_{2} n_{2}}{N_{2}}\right)\right), \quad n \in \Omega .
$$


In the expression above, $i$ denotes the imaginary unit. We further define the Discrete Fourier Transform (DFT) of $x$ as,

$$
\hat{x}(k)=\left\langle x, e_{k}\right\rangle=\sum_{n \in \Omega} x(n) \overline{e_{k}(n)}, \quad k \in \Omega .
$$

Here, $\bar{a}$ denotes the conjugate of a complex number $a \in \mathbb{C}$. Sometimes, we also use the notation $\hat{x}=\mathscr{F}\{x\}$ for the DFT.

Using the definitions above, it is straightforward to show that $\mathcal{C}(x) e_{k}=$ $x * e_{k}=\hat{x}(k) e_{k}$. In other words, the Fourier basis vector $e_{k}$ is an eigenvector of the convolution operation $\mathcal{C}(x)$ with the corresponding Fourier coefficient $\hat{x}(k)$ as its eigenvalue. Now, let $\mathcal{F}$ be the $N \times N$ Fourier basis matrix with its columns consisting of $\left\{e_{k}\right\}_{k \in \Omega}$. The DFT can then be expressed as $\hat{x}=\mathcal{F}^{\mathrm{H}} x$, where ${ }^{\mathrm{H}}$ denotes the conjugate transpose. Furthermore, it can be shown that the Fourier basis is orthogonal $\mathcal{F}^{\mathrm{H}} \mathcal{F}=N I$, giving us the inverse DFT $x=$ $\mathscr{F}^{-1}\{\hat{x}\}=\frac{1}{N} \mathcal{F} \hat{x}[31]$. The above results implies the following diagonalization of the convolution matrix,

$$
\mathcal{C}(x)=\frac{1}{N} \mathcal{F} \operatorname{diag}(\hat{x}) \mathcal{F}^{\mathrm{H}} .
$$

It turns out that the DFT has two truly remarkable properties. First, from (2.22) it follows that the eigenvectors of $\mathcal{C}(x)$ does not depend on $x$ itself. That is, any convolution matrix $\mathcal{C}(x)$ has the same set of eigenvectors $\mathcal{F}$. Second, there exists an algorithm with complexity $\mathcal{O}(N \log N)$ for computing the DFT $\hat{x}=\mathcal{F}^{\mathrm{H}} x$, famously known as the Fast Fourier Transform (FFT). Combined, it means that we can perform the diagonalization $(2.22)$ in $\mathcal{O}(N \log N)$ time.

The first immediate consequences of these results are the convolution theorems [31],

$$
\begin{aligned}
\widehat{x * f} & =\hat{x} \hat{f} \\
\widehat{x f} & =\frac{1}{N} \hat{x} * \hat{f} .
\end{aligned}
$$

Note that the multiplications above are performed point-wise. The result above implies that circular convolution can be performed in $\mathcal{O}(N \log N)$ instead of $\mathcal{O}\left(N^{2}\right)$ operations using the formula (2.23a). However, the consequences are even more important when it comes to solving equations involving convolutions. Consider the equation $x * f=y$ in $f$, which is equivalent to $\mathcal{C}(x) f=y$. Naively solving this in the spatial domain using e.g. Gauss elimination requires $\mathcal{O}\left(N^{3}\right)$ operations. But using (2.22), we can express the solution as $f=\mathscr{F}^{-1}\{\hat{y} / \hat{x}\}$, which only requires $\mathcal{O}(N \log N)$ operations.

As a final remark in this section, we shall study the differences between circular convolution and correlation. The circular correlation $x \star f$ between two real functions is defined by simply replacing the minus sign in (2.19) with a plus sign. Or equivalently, by replacing the filter $f\left(l_{1}, l_{2}\right)$ with 
its reflection $f\left(\left(-l_{1}\right)_{N_{1}},\left(-l_{2}\right)_{N_{2}}\right)$. As mentioned in the beginning of this chapter, correlation is more popularly used in the literature, thereby the name Discriminative Correlation Filters. Yet, from a machine learning perspective it does not mater which definition we use. In the next section, a method for learning the filter $f$ is presented. If we had chosen correlation instead of convolution, we would simply end up learning the reflected filter. The choice to use convolution in this thesis is motivated by its commutative property $x * f=f * x$, which leads to simpler formulas $(2.23)$.

\subsection{The MOSSE Tracker}

With the tools of linear regression, convolution and the Fourier Transform, we are now ready to introduce the MOSSE tracker. We have several times touched on the dataset $\mathcal{X}=\left\{\left(x_{j}, y_{j}\right)\right\}_{j=1}^{m}$ without detailing how it may be constructed. Again consider figure 2.2. We need to extract positive examples of the target appearance and negative examples of the surrounding background. Of course, one may employ different strategies here: random, grid-based, etc. In the generic tracking scenario however, training data is scarce, consisting of maybe only a single image. This suggests the inclusion of all relevant data. However, naively sampling all image patches in the target neighborhood would require considerable memory and computation for learning the regression parameters (2.17b).

The Minimum Output Sum of Squared Error (MOSSE) model by Bolme et al. [5] is able to utilize all information in the target neighborhood without suffering from the computational setback. In this case, let $x_{j}$ denote a larger image patch including both the target and the surrounding background, as visualized in figure 2.4. Further, let $y_{j}(n)$ denote the target score at location $n \in \Omega$ in the sample patch $x_{j}$. That is, both $x_{j}$ and $y_{j}$ are now functions $\Omega \rightarrow \mathbb{R}$. Typically, $y_{j}$ is set to a sampled Gaussian function with a narrow peak that is centered on the target, as in figure 2.4.

The aim of MOSSE is to find a filter $f$ such that $x_{j} * f \approx y_{j}$ for all samples $j$ in the dataset. But note that the convolution $x_{j} * f$ applies the weights in the filter $f$ to all possible cyclic shifts of the sample $x_{j}$. This very much resembles our initial desire of including every sample patch in the target neighborhood. Furthermore, by rewriting $x_{j} * f=\mathcal{C}\left(x_{j}\right) f$ it becomes apparent that this is indeed a linear regression problem. Each row in $\mathcal{C}\left(x_{j}\right)$ consists of a cyclicly shifted version of the original patch $x_{j}$. By concatenating all samples $j$ in the dataset, we get the data matrix $X$ and target vector $\mathbf{y}$, where

$$
X=\left(\begin{array}{c}
\mathcal{C}\left(x_{1}\right) \\
\vdots \\
\mathcal{C}\left(x_{m}\right)
\end{array}\right) \quad, \quad \mathbf{y}=\left(\begin{array}{c}
y_{1} \\
\vdots \\
y_{m}
\end{array}\right)
$$



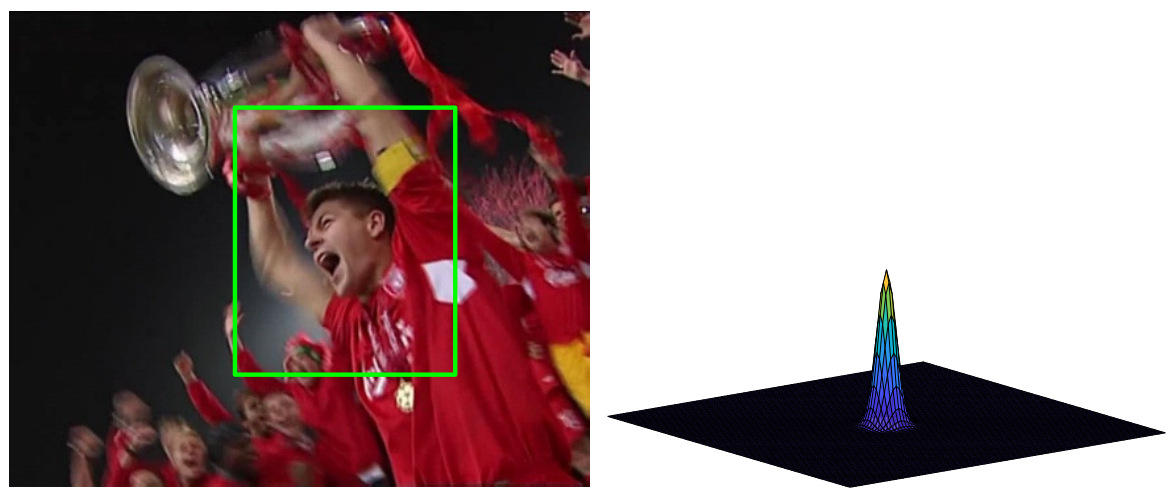

Figure 2.4: The image region corresponding to the sample patch $x_{j}$ in MOSSE (left, green box). Each pixel location $n$ in the region is given a target score $y_{j}(n)$ (right).

Now, we can directly apply $(2.17 \mathrm{~b})$ to compute the filter weights. For simplicity, first define $\lambda=\sigma^{2} / \nu^{2}$. Inserting (2.24) gives,

$$
f_{m}=\left(\sum_{j=1}^{m} \mathcal{C}\left(x_{j}\right)^{\mathrm{H}} \mathcal{C}\left(x_{j}\right)+\lambda I\right)^{-1} \sum_{j=1}^{m} \mathcal{C}\left(x_{j}\right)^{\mathrm{H}} y_{j} .
$$

Armed with the Fourier transform, we can diagonalize this problem using $(2.22)$,

$$
f_{m}=\frac{1}{N} \mathcal{F}\left(\sum_{j=1}^{m} \operatorname{diag}\left(\hat{x}_{j}\right)^{\mathrm{H}} \operatorname{diag}\left(\hat{x}_{j}\right)+\lambda I\right)^{-1} \sum_{j=1}^{m} \operatorname{diag}\left(\hat{x}_{j}\right)^{\mathrm{H}} \hat{y}_{j} .
$$

We can equivalently express the filter in terms of its Fourier coefficients by multiplying with $\mathcal{F}^{\mathrm{H}}$ from the left,

$$
\hat{f}_{m}=\frac{\sum_{j=1}^{m} \overline{\hat{x}_{j}} \hat{y}_{j}}{\sum_{j=1}^{m} \overline{\hat{x}_{j}} \hat{x}_{j}+\lambda} .
$$

The resulting formula is remarkably simple, containing only pointwise operations and DFTs. It is hence computed in $\mathcal{O}(N \log N)$ time by exploiting the FFT algorithm.

Another excellent feature of the formula (2.27) is that the filter can be easily updated with new training samples. Let us regard the sample index $j$ as the frame number. That is, sample $x_{j}$ was extracted from frame number $j$. In online learning, it is common to employ a learning rate parameter $\gamma \in[0,1]$ which controls the speed of which the model is adapted to the new data. Eq. (2.27) suggests updating the numerator $\hat{f}_{m}^{\text {num }}$ and denominator $\hat{f}_{m}^{\text {den }}$ of the filter $\hat{f}_{m}=\frac{\hat{f}_{m}^{\text {num }}}{\hat{f}_{m}^{\text {den }}}$ recursively as,

$$
\hat{f}_{m}^{\text {num }}=(1-\gamma) \hat{f}_{m-1}^{\text {num }}+\gamma \overline{\hat{x}_{j}} \hat{y}_{j} \quad, \quad \hat{f}_{m}^{\text {den }}=(1-\gamma) \hat{f}_{m-1}^{\text {den }}+\gamma\left(\overline{\hat{x}_{j}} \hat{x}_{j}+\lambda\right) .
$$



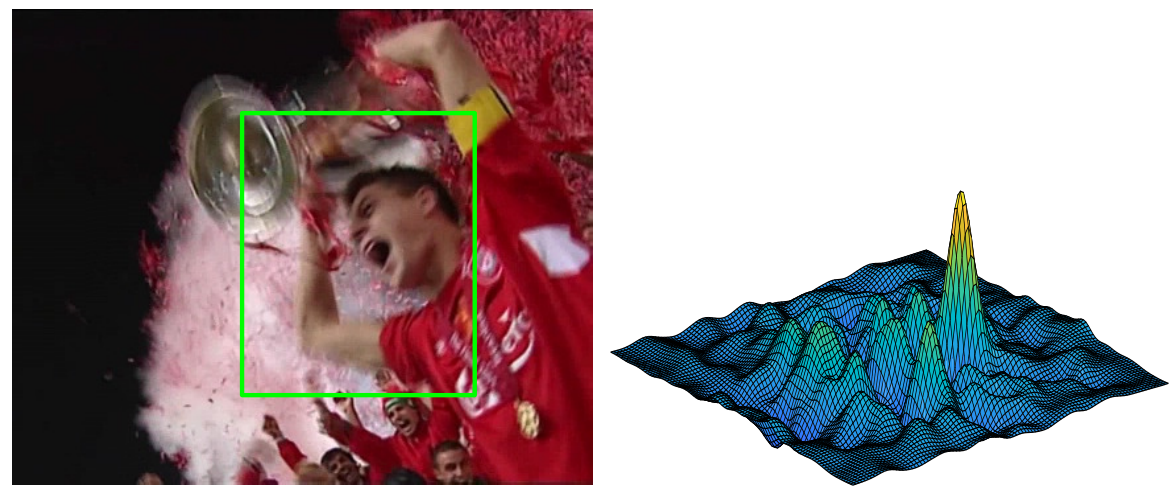

Figure 2.5: The image region (green box) corresponding to the image patch $z$ used for localizing the target in the given image (left). The score function $s$ (right), obtained by applying the learned filter to $z$, contains the predicted target score $s(n)$ for each pixel location $n$ in the patch $z$.

In fact, this update scheme finds the filter $\hat{f}_{m}$ that minimizes the weighted squared error,

$$
L(f)=\sum_{j=1}^{m} \alpha_{j}\left\|x_{j} * f-y_{j}\right\|^{2}+\lambda\|f\|^{2} .
$$

Relating back to (2.17), we can conclude that the sample weights $\alpha_{j}>0$ are inversely proportional to the variance of the target values $y_{j}$. To achieve the solution given by the recursive formula (2.28), we must choose the weights $\alpha_{j}=\gamma(1-\gamma)^{m-j}$ for $j>1$ and $\alpha_{1}=(1-\gamma)^{m-1}$.

Next, we shall se how the MOSSE filter can be used to efficiently localize the target in a new frame. Let $m$ be the number of the current image frame, in which we wish to localize the target. From the previous frame we are given the filter $\hat{f}_{m-1}$, that has been recursively updated since the initial frame. We extract an image patch $z$ centered at the predicted target location, as depicted in figure 2.5. Here, $z$ has the same size $N_{1} \times N_{2}$ as the training patches $x_{j}$. Using the convolution theorem (2.23a), we then predict target scores $s(n)$ at each location $n \in \Omega$ in $z$ by applying the learned filter,

$$
s=z * f_{m-1}=\mathscr{F}^{-1}\left\{\hat{z} \hat{f}_{m-1}\right\} .
$$

This computes the score function (2.14) and the mean (2.18) of the predictive distribution at all locations $n \in \Omega$. We can then estimate the target location in frame $m$ as the maximizer of the target score function $n^{*}=\arg \max _{n} s(n)$. If we are confident about our estimate, we may construct a new training sample $\left(x_{m}, y_{m}\right)$ by extracting a patch $x_{m}$ centered at the estimated location $n^{*}$. Finally, the filter is updated by applying (2.28).

Lastly, we compute the entire posterior predictive distribution $p(y \mid z, \mathcal{X})$ (2.18) for completeness. As previously noted, the target score $s(n)$ in $(2.30)$ is 
the expectation of this distribution location $n$. But we are yet to evaluate the variance. Since the predictions are performed by a circular convolution with the learned filter weights $f$, the actual sample that is evaluated at location $n$ is a reflected and cyclicly shifted version of the image patch $z$. We start by evaluating the variance $\sigma^{2}+z^{\mathrm{T}} V_{m} z$ of the non-translated sample $z$ itself. A similar computation to $(2.26)$ yields,

$$
z^{\mathrm{T}} V_{m} z=\frac{\sigma^{2}}{N}\left(\mathcal{F}^{\mathrm{H}} z\right)^{\mathrm{H}}\left(\frac{\hat{z}}{\sum_{j=1}^{m} \hat{x}_{j} \hat{x}_{j}+\lambda}\right)=\frac{\sigma^{2}}{N} \sum_{k \in \Omega} \frac{|\hat{z}(k)|^{2}}{\sum_{j=1}^{m}\left|\hat{x}_{j}(k)\right|^{2}+\lambda} .
$$

Note that the right hand side only depends on the amplitude $|\hat{z}(k)|$ but not the phase $\arg \hat{z}(k)$ of the DFT. A well known property of the DFT, directly verifiable from the definition (2.21), is that cyclic shifts of the original function $z$ only affects the phase but not the amplitude. The variance $(2.31)$ is thus independent of the translation of $z$. The reflection induced by the convolution does not change the conclusion since it can be applied prior to the translation of the image patch $z$. As a consequence, the variance of the score $s(n)$ is the same at every location $n$ in $z$, and therefore of lesser use.

To summarize this chapter, we started off with a simple generative model $p(x)$ of the target appearance. This model was used to derive the famous Lucas-Kanade and NCC techniques. Then, we saw how background information can be incorporated by augmenting the generative model with a binary variable $y$ indicating the presence of the target. This approach was then modified into a discriminative model by directly optimizing the decision criterion on the training set. However, the resulting model inference relies on non-linear optimization, less suitable for the online tracking scenario. We therefore reintroduced Gaussianity by transforming the output $y$ to a continuous variable indicating the target confidence score. Finally, we introduced circular convolution and used the properties of the discrete Fourier transform to derive efficient model inference and prediction formulas. In the next chapter, we build upon these ideas in the development of the Discriminative Correlation Filter framework. 


\section{Discriminative CORRELATION FILTERS}

This chapter gives an overview of the DCF tracking framework, that is the main subject of this thesis. It is organized into different sections, each presenting a different aspect of the DCF. The intention is to provide an overview, as well as to motivate and introduce the contributions of the included publications. The reader is then referred to the papers themselves for a detailed treatment.

\subsection{The Kernelized DCF}

As we will see in this chapter, there are numerous limitations of the basic MOSSE tracker presented in section 2.8, many of which are addressed in this thesis. Arguably, the most important of these is the image representation it employs. In the previous chapter, we assumed the sample patches $x$ to consist of the grayscale image intensities. Except for the simplest cases, such a representation is too simplistic for visual object tracking when employing a linear model. To alleviate this issue, one solution that comes to mind is the integration of more powerful feature representations. That is, we construct some non-linear mapping $\phi(x)$ which extracts discriminative image features that are more suited for the target recognition problem. However, such powerful representations are often high-dimensional: $\phi(x)(n) \in \mathbb{R}^{D}$, whereas we in section 2.8 assumed the sample patch to be one-dimensional $x(n) \in \mathbb{R}$. Later, we will study how multidimensional features can be directly incorporated into the MOSSE formulation. But first, we will visit an alternative approach.

An early extension of the MOSSE formulation was the introduction of kernels by Henriques et al. [42, 41]. Kernels is a popular machine learning technique that can be applied in a variety of methods including linear 
regression $[70,75]$. The basic idea is to introduce a similarity measure $\kappa\left(x, x^{\prime}\right)$ between two samples $x$ and $x^{\prime}$ that implicitly defines a non-linear mapping $\phi$ by the relation $\kappa\left(x, x^{\prime}\right)=\left\langle\phi(x), \phi\left(x^{\prime}\right)\right\rangle$. That is, $\kappa$ defines the inner product between two samples in some high-dimensional (often infinite) feature space $\phi(x)$. By exploiting the kernel trick [70, 4], the feature mapping $\phi$ never needs to be explicitly constructed.

In literature, one of the most popular kernels is the Gaussian radial basis function (RBF) kernel $\kappa\left(x, x^{\prime}\right)=\exp \left(\frac{1}{\sigma^{2}}\left\|x-x^{\prime}\right\|^{2}\right)$. In addition to the necessary conditions of being symmetric and positive definite [4], this kernel cyclicly shift invariant [41]. That is, it has the property $\kappa\left(\tau_{l} x, x^{\prime}\right)=\kappa\left(x, \tau_{-l} x^{\prime}\right)$, where $\left(\tau_{l} x\right)(n)=x\left((n-l)_{N}\right)$ is the cyclic shift operator. This property preserves the circulant structure of the MOSSE formulation, ensuring that the DFT can be applied in a similar manner to efficiently infer the appearance model. The reader is referred to $[41,10]$ for a more detailed treatment on the integration of kernels.

The kernel formulation however introduces an issue. While the MOSSE tracker solution (2.27) exhibits a linear complexity in the number of training samples $m$, the complexity for the kernelized variant is cubic $\mathcal{O}\left(m^{3}\right)$. This is a well known fact in Gaussian Process regression, which is strongly related to linear regression with kernels [75]. To overcome this issue, Henriques et al. [42] proposed to solve for a single training sample at a time, and update the model parameters using a simple learning rate. However, such a strategy is only an ad-hoc integration of new information, leading to a suboptimal appearance model.

In Paper A, an alternative kernelized model is proposed that preserves the original $\mathcal{O}(m)$ complexity of the MOSSE. This is achieved by constraining the model parameters to be shared for all training samples, achieving a principled approximation of the ideal loss. The new formulation allows a simple update rule, similar to the one of MOSSE (2.28). This model update is shown to be more robust for a large set of different color features.

\subsection{Multidimensional Feature Maps}

As mentioned in the previous section, a powerful feature representation is essential in order to tackle the general object tracking problem. In this section, we study how to integrate multidimensional features into the DCF formulation. In the previous chapter, we considered $x$ to be a grayscale image patch for simplicity. The immediate question that arises is how we can exploit the color information encoded in RGB-images. To represent an RGB-patch, we need a vector valued function $x: \Omega \rightarrow \mathbb{R}^{3}$ where each pixel $n$ is represented by a 3 -dimensional RGB vector $x(n) \in \mathbb{R}^{3}$.

In general however, $x$ could be any representation of the image defined over the spatial domain $\Omega$. For example, $x$ can be formed by converting the 
image to another color space, computing the image gradients or applying a deep convolutional neural network. Different feature representations will be discussed in more detail in chapter 4 . We call $x: \Omega \rightarrow \mathbb{R}^{D}$ a feature map, since it maps every spatial location $n$ to a $D$-dimensional feature vector $x(n) \in \mathbb{R}^{D}$.

However, $x$ cannot be any representation or mapping of the original image. It needs to be spatially invariant. That is, the feature mapping process should be independent of the location where it is applied. Formally, let $J$ be the input image and $x=\phi(J)$ be the resulting feature map. Then, translating the feature map $x(\cdot-l)$ by $l$ steps should correspond to translating the original image by $r \cdot l$ pixels. That is, the function $\phi$ must satisfy $x(\cdot-l)=\phi(J(\cdot-r l))$. The factor $r \in \mathbb{N}$ is called the stride of the feature map. It corresponds to the amount of spatial downsampling that is performed in the feature extraction step. It is easy to see why spatial invariance is important in the tracking scenario. When searching for the target in the image, we need to count on the feature representation of the target appearance not to depend on where the target might hide in the image. This enables us to search for the target over the entire image using the same model, whether generative, dicriminative, linear or non-linear.

Now, let us extend the MOSSE model (2.29) to the case of multidimensional feature maps. We introduce the notation $x^{d}$ for the $d$-th feature channel $x^{d}: \Omega \rightarrow \mathbb{R}$, representing the $d$-th dimension in the feature map. Similarly, we extend the filter $f$ to be $D$-dimensional, denoting the individual filter channels with $f^{d}$. The convolution between two multidimensional functions is defined by simply summing the convolution responses for each individual channel. We can thus learn the filter $f$ by minimizing,

$$
L(f)=\sum_{j=1}^{m} \alpha_{j}\left\|\sum_{d=1}^{D} x_{j}^{d} * f^{d}-y_{j}\right\|^{2}+\lambda \sum_{d=1}^{D}\left\|f^{d}\right\|^{2} .
$$

This multichannel formulation above, which forms the basis of DCF, is still a linear least squares problem. It cannot be fully diagonalized by the DFT in the general case. There are however two exceptions, where diagonal solutions are found. We have already studied the case $D=1$, which corresponds to the original MOSSE, allowing the simple formula (2.27). The other case occurs when there is only a single training sample $m=1$, resulting in the following optimal filter,

$$
\hat{f}^{d}=\frac{\overline{\hat{x}^{d}} \hat{y}}{\sum_{l=1}^{D} \overline{\hat{x}^{l}} \hat{x}^{l}+\lambda} .
$$

This result is proved in the appendix of Paper C. In principle, the proof utilizes the dual form [70] of the general solution $(2.17 \mathrm{~b})$. It can also be derived by applying the linear kernel to the kernelized solution in [41]. In Paper C, the solution (3.2) above is used to derive a simple update rule for the multichannel 
case that reduces to the optimal filter solution in both the cases of a single sample $m=1$ and a single feature channel $D=1$.

Even though no simple diagonal solution can be achieved in general by applying the DFT to (3.1), it still drastically reduces the computational complexity of a solver. Using (2.17b) and the techniques described in section 2.8, the problem (3.1) can be rephrased as solving a linear equation system of the form $A \hat{f}=b$, called the normal equations. Here, $\hat{f} \in \mathbb{C}^{D N}$ is a vectorization of all DFT coefficients $\hat{f}^{d}$ of $f, A$ is a $D N \times D N$ Hermitian positive-definite matrix, and $b \in \mathbb{C}^{D N}$ is a vector. In fact, it can be shown [29] that $A$ exhibits a block-diagonal structure, with one $D \times D$ block for each DFT frequency $k$. The total computational complexity for a direct solver is thus $\mathcal{O}\left(D N \log N+N D^{3}\right)$, while a direct solution in the spatial domain requires $\mathcal{O}\left(D^{3} N^{3}\right)$ operations. As we will see in section 3.8, iterative solvers such as Gauss-Seidel [37] and Conjugate Gradient [82] are more practical for the online tracking scenario.

Multidimensional feature maps can also be incorporated in the kernelized formulation of DCF, introduced in section 3.1. This is utilized for the investigation of different color features in Paper A. Later studies $[41,10]$ have indicated that similar or better performance can be obtained with the standard linear kernel. Furthermore, the standard linear formulation (3.1) is advantageous in terms of computational and memory complexity. Therefore, only Paper A and Paper B employ the kernelized DCF formulation, while all subsequent papers in this thesis reside to the linear version.

\subsection{Scale Estimation}

As we have seen, translations are at the heart of the DCF methodology. The convolution slides the filter over the image patch, searching for the target at different locations. Furthermore, the discrete Fourier transform diagonalizes this operation. This seems a satisfactory model of the tracking scenario as long as the target is only moving parallel to the image plane. But if the target moves towards or away from the camera, its apparent size in the image changes. This is known as a scale transformation.

There are primarily two reasons why estimating the scale of the target is important. First, in many applications, such as robotics, the distance to the target is of interest. According to the pin-hole camera model [39], the relative distance to the target is in fact inversely proportional to the target size in the image. Estimating the scale thus provides information about the distance. Second, failure in estimating the target scale often leads to severe model drift. An example is illustrated in figure 3.1. The red box is the output of a tracker with scale estimation, while the green box does not estimate scale at all. When the target is moving away from the camera, more background regions are incorporated into the tracking model. Eventually, the non-scale 

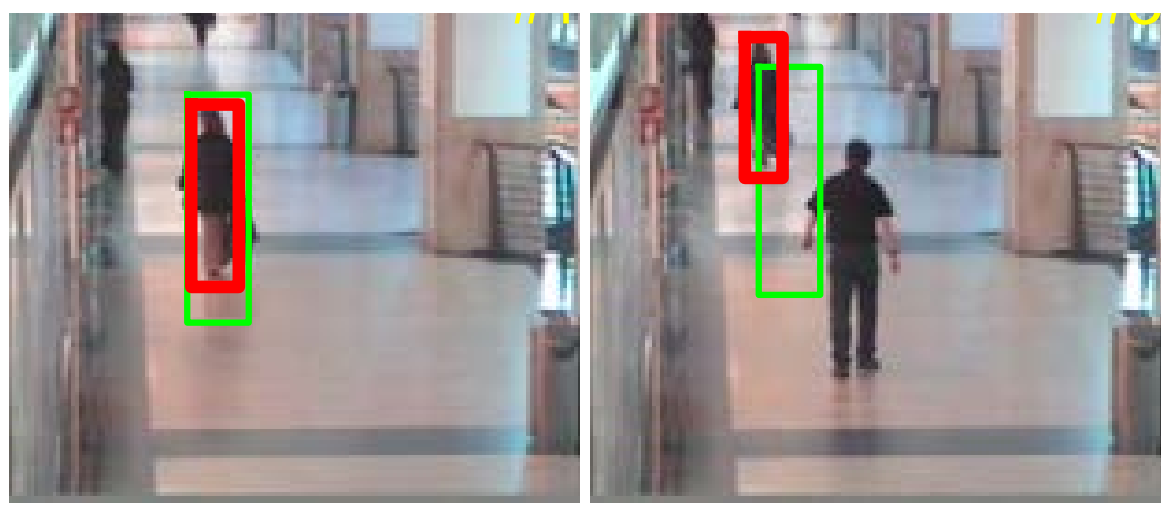

Figure 3.1: Illustrating the importance of scale estimation. The scale adaptive tracker (red box) successfully tracks the woman in the video. On the other hand, the non-scale adaptive tracker (green box) experiences severe drift as the model is corrupted with background information.

tracker drifts off the target as its model gets too corrupted with background data. The scale-adaptive tracker successfully continues to track the target.

Paper $\mathrm{C}$ investigates different alternatives for incorporating scale estimation into the DCF framework. One straight-forward approach is to apply the learned translation filter $f$ at different image scales. That is, the image is first resized by different scale factors, followed by feature extraction. The feature map at each scale is then convolved with the learned filter $f$ to compute the target scores. The change in target location and scale can then be estimated by finding the maximum score across all scales. This is a common strategy, often applied in both visual object tracking and detection [28].

In Paper C, an alternative strategy is suggested, initially proposed by the authors in [13]. To avoid an exhaustive search across translation and scales, target estimation is performed in two steps. Since the scale change between two frames is usually small or moderate, the target translation is first found by applying the normal translation filter at the current scale estimate. Then, a separate one-dimensional filter is applied in the scale dimension to update the target size. The scale filter is trained analogously to the translation filter, but operates in the scale dimension by extracting samples of the target appearance from a set of different scales.

The advantage of the proposed scale-filter approach is two-fold. Computational efficiency is gained by reducing the search space. Further, the scale filter is trained to discriminate between the appearance of the target at different scales, which can lead to more accurate estimation. The proposed scale filter component has been applied in a multitude of trackers in literature, 
including $[44,65,64,3]$, and was a part of the author's winning contribution [13] to the Visual Object Tracking (VOT) 2014 challenge [54].

Later improvements of the translation filter model, discussed in the subsequent sections, and more powerful deep feature representations have renewed the interest for the multiscale translation filter methodology. But thanks to its computational advantage, the scale filter is still of interest. It is used in the faster version of the ECO tracker presented in Paper I, which is the most recent contribution of this thesis.

\subsection{Periodic Assumption and Spatial Regularization}

So far, we have generalized the DCF framework to multidimensional feature representations and included the ability of scale estimation. This motivated us to tackle another important limitation of the DCF, having severe implications on tracking performance and applicability. In the previous chapter, we studied linear regression and derived the equation (2.14) that generates target predictions across the image. We concluded that this equation corresponds to a linear correlation between the image $J$ and the regression weights $f$. Of course, it could be restated as a convolution by just reflecting the filter $f$. While motivating the MOSSE tracker in section 2.8, the standard convolution was effectively replaced by circular convolution to ensure the applicability of the DFT, resulting in the formula (2.30) for evaluating the predictions. This change may seem minor and might even have gone unnoticed to some readers. But as we will see next, the circular convolution is a severe limitation, which rivals the previously addressed single-channel assumption in importance.

To investigate the impact of circular convolution, we must first understand its definition (2.19). What differentiates circular from standard convolution is the modulo operation in the expression $x\left(\left(n_{1}-l_{1}\right)_{N_{1}},\left(n_{2}-l_{2}\right)_{N_{2}}\right)$. That is, when the coordinate $n-l$ goes outside the domain of $x$ it is wrapped around. To visualize the wrap-around effect, we can equivalently think of $x$ as periodically extended outside its original domain $\Omega$. This is visualized in figure $3.2 \mathrm{a}$. That is, circular convolution $x * f$ can equivalently be computed by first forming the periodic extension $\tilde{x}$ of the image patch $x$ and then applying ordinary convolution with the filter $f$,

$$
x * f(n)=\sum_{l \in \Omega} \tilde{x}(n-l) f(l), \quad n \in \Omega .
$$

Let us compare the periodic extension $\tilde{x}$ in figure $3.2 \mathrm{a}$ with the corresponding actual image patch of the same size (right). The vast majority of the background pixels are replaced by the periodic repetitions. It is apparent that the periodically repeated patch only serves as a very crude approximation of the original image content. The question is how this periodic approximation of the image content, induced by circular convolution, affects the DCF tracker. 


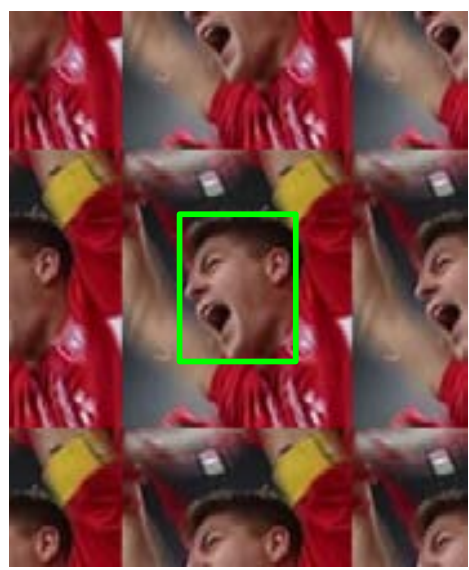

(a) Periodic repetition.

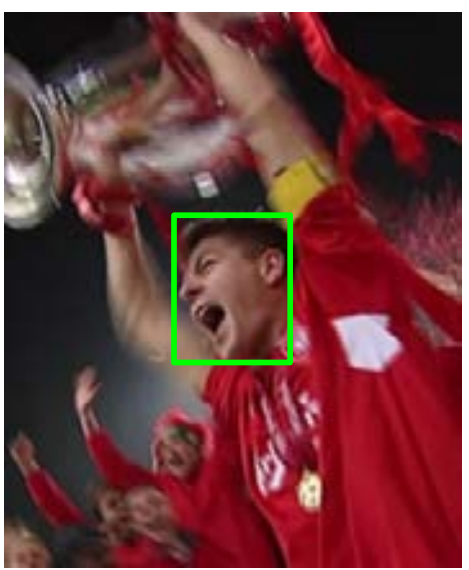

(b) Original image sample.

Figure 3.2: Visualization of the periodic effects caused by the circular convolution. The sample patch is synthetically extended using periodic repetitions of the smaller patch (a). This is only a harsh approximation of the original image content (b).

The most severe consequence of the circular convolution assumption arises in the learning formulation (3.1). The fundamental idea in the DCF paradigm is to train a filter $f$ that can discriminate the target from background imageregions. But due to the periodic effects, most of the original background content (figure $3.2 \mathrm{~b}$ ) is replaced by synthetic repetition of a smaller image patch (figure 3.2a). The model thus sees less background samples during training, severely limiting its discriminative power. Furthermore, due to the distortions caused by the periodic repetitions, the predicted target scores are only accurate near the center of the image patch. The size of the search area is therefore limited.

As traditionally performed in signal processing, DCF methods typically pre-process the samples $x$ by multiplying them with a window function [5]. But this technique does not attempt to solve the aforementioned problems, only serving as a bandaid to smooth out the discontinuities at the border regions. A naïve solution is instead to simply extend the original image patch $x$ to cover a larger part of the image. Indeed, the periodic effects are reduced by simply increasing the period of repetition, thereby including more true image data. But the filter and sample sizes are tied in the DCF, requiring a similar increase of the filter size. As a consequence, the positive target samples are diluted with background information. Furthermore, the filter is trained to track the background as much as the foreground (see figure 3.3a). This effectively forces a small sample size to be used during training. 


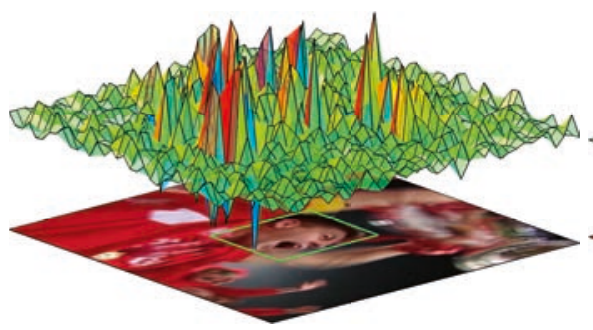

(a) Standard DCF.

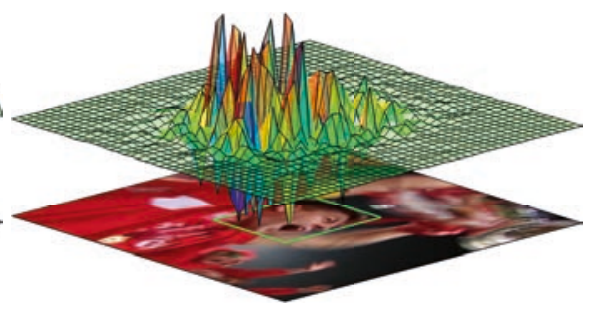

(b) Spatially regularized filter.

Figure 3.3: Comparison between the filter coefficients learned using the standard DCF formulation (a) and the spatially regularized filter (b). The figures visualize the filter coefficients $f^{d}$ of a feature channel $d$ and the corresponding image region used for training. In the standard DCF, high filter coefficients are learned for the background region, reducing its discriminative power. The spatial regularization weights $w$ penalize filter values corresponding to features in the background, emphasizing the appearance information within the target region (green box).

The problem of enlarging the sample size arises due to the absence of a mechanism for independently controlling the filter extent. This key limitation of the DCF framework is addressed in Paper D. A spatial regularization component is augmented to the learning formulation,

$$
L(f)=\sum_{j=1}^{m} \alpha_{j}\left\|\sum_{d=1}^{D} x_{j}^{d} * f^{d}-y_{j}\right\|^{2}+\sum_{d=1}^{D}\left\|w f^{d}\right\|^{2} .
$$

The spatial weight function $w: \Omega \rightarrow \mathbb{R}$ takes positive values $w(n)>0$, penalizing the filter coefficients $f^{d}(n)$ based on their spatial location $n$. By letting $w$ take large values at background pixels and small values inside the target region, background filter coefficients are penalized. As a result, a compact filter $f$ focusing on the target region can be learned, even for large image sample sizes. The difference between a filter trained with and without spatial regularization is visualized in figure 3.3.

According to the Bayesian interpretation presented in section 2.6, the spatial regularization corresponds to a prior $p(f)=\mathcal{N}\left(0, \operatorname{diag}\left(w^{-2}\right)\right)$ on the filter coefficients. That is, the filter coefficient $f(n)$ has a zero-mean Gaussian prior with variance $w(n)^{-2}$. Thus, a larger regularization value $w(n)$ corresponds to a stronger belief that $f(n) \approx 0$. We can use $w$ to encode our prior knowledge about the extent of the target object, and thereby the filter itself.

The challenge comes when trying to find a tractable inference algorithm, minimizing the loss (3.4), for the online tracking application. The main question is if we can still apply the DFT to simplify the optimization problem. As we know from the first convolution theorem (2.23a), the convolution in 

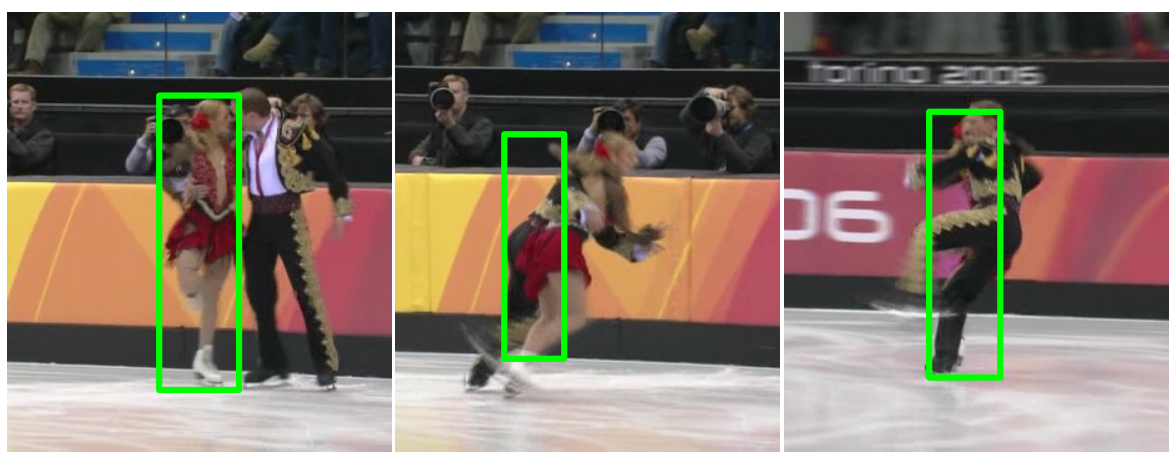

Figure 3.4: Visualization of corrupted samples collected during tracking. Tracking is initialized in the left image, where the aim is to track the female figure skater. In the center frame, the tracking prediction is misaligned due to the complex appearance changes of the target. In the right frame, the target is occluded by the male skater. In both aforementioned cases, the collected training samples are erroneous as they are annotated by the location predicted by the tracker itself.

(3.4) diagonalized to a point-wise multiplication. But according to the second convolution theorem $(2.23 \mathrm{~b})$, the product $w f^{d}$ in the regularization term transforms into a convolution $\hat{w} * \hat{f}^{d}$. It seems that the benefit of applying the DFT is gone, since its purpose was to remove the convolution operations in the loss.

One of the key insights presented in Paper D is that the regularization weights $w$ can be constructed using only very few DFT coefficients $\hat{w}$. The corresponding convolution matrix $\hat{w} * \hat{f}^{d}=\mathcal{C}(\hat{w}) \hat{f}^{d}$ therefore has a very sparse structure. Although the resulting normal equations are no longer block-diagonal, they still retain most of their sparsity. This key observation paves the way for iterative solvers to be applied in the online learning case. Experimental evaluations in Paper D demonstrate the remarkable impact of the spatial regularization on the robustness of the tracker. The proposed spatial regularization has therefore remained a core component in all subsequent tracking papers by the author.

\subsection{Adaptive Training Set Management}

In the previous section, we studied how the circular convolution assumption inherent in DCF corrupts the training data with periodic repetitions. The spatial regularization component was introduced to alleviate this issue by allowing the sample size to be increased. There are however other factors that contribute to the inclusion of corrupted training data. As previously noted, one of the major challenges of generic visual tracking is the lack of 
training data. It is therefore tempting to harvest additional training samples during tracking. But as no other source of information is available, the tracker must annotate the new samples itself. The correctness of these annotations is directly related to the accuracy of the tracking predictions.

Tracking predictions may be inaccurate or incorrect due several reasons. For example, this may occur if the target undergoes out-of-plane rotations or complex 3D-deformations, in turn leading to misaligned annotations of the collected training samples. Including such samples in the model update can lead to model drift and eventually complete tracking failure. Furthermore, when the target is occluded, positive training samples are contaminated by background structures or other objects in the scene. Such samples reduce the discriminative power of the appearance model when included in the training set. These two issues, that are visualized in figure 3.4, affects all tracking approaches that attempt to update the appearance model online.

Paper F directly addresses this fundamental problem in visual tracking. The core idea of the paper is to jointly optimize the model parameters, i.e. the filter $f$, and the sample importance weights $\alpha_{j}$ in (3.4). The importance $\alpha_{j}$ of the sample $x_{j}$ at frame $j$ is determined during the model inference procedure. Corrupted samples are often outliers, exhibiting a comparatively large loss. Our formulation forces their corresponding weights $\alpha_{j}$ towards zero, effectively removing them from the training set. In addition, prior knowledge about the weights $\alpha_{j}$ is incorporated by adding a regularization term.

The approach proposed in Paper $\mathrm{F}$ is to the best of our knowledge the first unified learning formulation jointly inferring the tracking model and the sample weights. It is applicable to a large class of discriminative models, and not restricted to DCF. In Paper F, it is experimentally evaluated with both a DCF-based and an SVM-based tracking model. The results show that tracking performance is significantly improved, especially in challenging tracking scenarios where corrupted samples are common.

\subsection{Continuous Formulation}

Previously, in section 3.2, we generalized the MOSSE tracker to multidimensional feature maps. This was done with a subtle restriction, namely that all dimensions in the feature map have the same spatial resolution $N_{1} \times N_{2}$. This assumption certainly holds for many classical feature representations, such as Histogram of Oriented Gradients (HOG) [9] and common color representations. But in recent years, deep features extracted from Convolutional Neural Networks (CNNs) have demonstrated superior performance in numerous computer vision tasks, such as classification [40], detection [77] and semantic segmentation [90]. Furthermore, these learned features have shown to be surprisingly generic and can be applied to other 
tasks. This motivated us to investigate how to integrate deep feature maps into DCF-based visual tracking.

Deep features are discussed in more detail in section 4.4, but we will summarize the most important concepts here. CNNs consist of a number of convolutional layers. The initial layers extract low-level features, such as edges and colors, at a high spatial resolution. The activations from deeper layers typically represent more high-level information, such as object parts. But due to pooling layers and strided convolutions, deeper feature maps typically have a lower spatial resolution yet higher dimensionality. As shown in Paper E, activations from early convolutional layers are important for tracking, as they contribute to localization accuracy. On the other hand, deeper layers are expected to provide robustness due to their high-level invariance to appearance changes. This raises the question of how to fuse early and late convolutional features in the DCF framework to benefit from their complementary properties.

Now, the limitation of a single-resolution feature map in DCF becomes problematic. Deep convolutional features extracted from different layers in the network generates a multiresolution feature map in general. That is, the spatial resolution of the extracted features varies across the dimension. In general, we let the resolution of feature channel $x^{d}$ in the sample $x$ be $N_{1}^{d} \times N_{2}^{d}$. Integrating such feature maps into the DCF formulation (3.4) is not possible since the resolution of the different feature channels do not match up.

In Paper $\mathrm{H}$ this limitation is addressed by introducing a novel continuousdomain formulation of the DCF framework. An implicit interpolation model is used to map the multiresolution features to the continuous spatial domain. We then learn a set of continuous-domain filters, parametrized by their Fourier coefficients. Since both the feature map and the filters are defined over the same continuous spatial domain, the convolution operation can be performed jointly across all feature dimensions. The resulting target score function is thus also defined over the continuous spatial domain. We show that all operations can be performed in the Fourier domain, completely avoiding explicit re-sampling or interpolation of features.

The proposed continuous formulation enables a natural integration of multiresolution features. Moreover, it provides a principled way of performing sub-pixel localization and learning. In section 2.1 we briefly noted that the Lucas-Kanade tracker can perform sub-pixel localization by continuous optimization and explicit re-sampling of the image. In Paper $\mathrm{H}$ we show that sub-pixel localization can indeed be achieved in our approach by maximizing the continuous target scores. Furthermore, the tracking model can be updated using sub-pixel supervision, thereby avoiding explicit interpolation of the training sample. Since these are desired properties for feature-point tracking, we also evaluate our approach for this application and compare with the LucasKanade algorithm. 
Paper I further builds upon the continuous learning formulation proposed in Paper H. The primary focus of Paper I is to reduce the computational complexity of the tracker, making it suitable for real-time applications. Furthermore, it is shown that this reduction in complexity also increases the robustness as the risk of overfitting is reduced. In particular, a factorized convolution operation is introduced, discussed in the next section. Additionally, a compact representation of the training set is proposed and better heuristics for the model update strategy are investigated.

\subsection{Dimensionality Reduction}

Keeping the computational cost at a minimum is one of the major challenges in generic visual tracking. It is also particularly important as most tracking applications demand real-time processing. The introduction of multidimensional feature maps increases the complexity of the model inference and target localization steps. As mentioned in section 3.2, the complexity of a direct solver applied to (3.1) scales cubically in the feature dimension $D$. The inference methods proposed in this thesis, based on approximative update rules or iterative optimization, exhibit linear or quadratic complexity in $D$. Hence, the number of feature dimensions plays a central role in the overall computational complexity of the tracking algorithm.

In general, we want the tracker to be generic, meaning it should be applicable to a wide range of scenarios, targets, backgrounds and cameras. It is therefore important to employ a generic feature representation that can cope with such variations. But a generic representation may yield many redundant features for the particular tracking task. For a specific video, only a minority of the feature channels might suffice to discriminate between the target and background. In this section we discuss approaches to learn a subset of discriminative features online. This task is commonly known as dimensionality reduction.

In order for a dimensionality reduction technique to be applicable, the computational overhead required in finding and performing mapping must be small compared to the computations saved by reducing the dimensionality. In this thesis, we therefore limit our attention to linear techniques. Among these, Principal Component Analysis (PCA) [4] is one of the most common. Geometrically, it finds the linear subspace that accounts for most of the variance in the features. The projection matrix $P$, mapping the features to this subspace, is found by simply performing an eigenvalue decomposition of the feature covariance matrix. The columns of $P$ are set to the $D^{\prime}<D$ eigenvectors corresponding to the largest eigenvalues. A new feature map $\tilde{x}$ of dimension $D^{\prime}$ is then constructed by the linear projection $\tilde{x}(n)=P^{\mathrm{T}} x(n)$.

In the tracking scenario, the feature statistics of the entire video is not initially available as it often changes over time. However, adapting the feature 
subspace, defined by $P$, based on new data is problematic. If the mapping $P$ is updated too rapidly, the already learned appearance model becomes obsolete, requiring expensive retraining and storage of old feature maps. In Paper A the standard PCA is extended with a temporal smoothness term, penalizing deviations from the previous subspaces. This ensures a robust adaption of the projection matrix $P$ to new data while not rendering the previously computed model parameters obsolete. A simplified variant of this approach, designed specifically for linear kernels is proposed in Paper C.

Relating back to the discussion in section 2.3, PCA can be understood as a generative approach for finding the projection matrix $P$. It is purely based on the statistics of the data samples $x$. Therefore, it does not necessarily find the feature mapping $P$ that is optimal for the task of discriminating the target from the background. Indeed, a feature channel $x^{d}$ could have low variance overall, yet still produce a distinctive signature at the target location. To find such distinctive features, a better alternative is to discriminatively learn the linear mapping $P$.

In Paper I, the matrix $P$ and filter $f$ are learned in a joint fashion by minimizing the discriminative loss corresponding to (3.4). Together, they form a factorized convolution operator $f * P^{\mathrm{T}} x$, where the sample is first point-wise multiplied by the weight matrix $P^{\mathrm{T}}$ and then convolved with a smaller set of $D^{\prime}$ filters $f$. Unfortunately, the resulting loss function does no longer correspond to a linear least-squares problem. In the paper, a Gauss-Newton based optimization procedure is therefore proposed, where Conjugate Gradient is applied for the quadratic subproblems. The introduced factorized convolution drastically reduces the number of feature dimensions $D$, in particular when using deep features. As demonstrated in Paper I, this does not only speed up the tracker, but also improves performance by reducing overfitting.

\subsection{Optimization}

Finally, in this chapter, we will dwell into how to infer the DCF model given a set of training samples $\left\{\left(x_{j}, y_{j}\right)\right\}_{j=1}^{m}$. In the initial MOSSE filter case, presented in section 2.8, inference was performed by computing the DFT coefficients $\hat{f}$ using the simple formula (2.27). Now, the model has grown more complex, by e.g. introducing multiresolution feature maps and spatial regularization. These modifications require alternative inference methods. However, as mentioned in the previous section, computational efficiency is a crucial factor in most tracking application. Therefore, finding efficient and robust inference algorithms is a key problem in DCF-based tracking.

Model inference is performed by minimizing the loss (3.1), which forms the basis of the DCF framework. However, since it does not allow any simple closed-form solution, many DCF trackers employ the diagonalizable cases 
(2.27) or (3.2) to derive approximate model inference schemes. These formulas rely on the very restrictive assumptions of a single feature channel $D=1$ and a single training sample $m=1$ respectively. Moreover, these solutions cannot benefit from the spatial regularization, introduced in (3.4). Thus, there is a strong need for principled, yet efficient methods for minimizing the loss (3.4).

Minimizing the DCF loss (3.1) or its spatially regularized variant (3.4) online is a highly challenging problem. Depending on the employed feature representation, the filter $f$ contains tens or hundreds of thousand parameters to be optimized. Therefore, solvers need to be tailored to the particular structure of the optimization problem. This can be done by exploiting two key characteristics of (3.4). First, the resulting normal equations exhibit a particular sparsity structure after transforming the problem to the Fourier domain. Second, the optimal filter $f$ is expected to change slowly when new training samples $x_{j}$ are added during tracking. Therefore, the current estimate of the filter $f$ serve as a good initialization point for the optimization, reducing the time required to find a new optimum.

In Paper D, an optimization approach based on the Gauss-Seidel algorithm [37] is proposed to minimize the spatially regularized loss (3.4). First, the normal equations are transformed to a real Fourier basis to preserve the number of degrees of freedom present in the spatial-domain formulation (3.4). As a result, the normal equations $\tilde{A} \tilde{f}=\tilde{b}$ form a real equation system, where $\tilde{f}$ is the transformed filter coefficients, $\tilde{b}$ is a real vector and $\tilde{A}$ is a real, symmetric and positive definite matrix that is sparse. The Gauss-Seidel algorithm decomposes the matrix $\tilde{A}$ into the sum of a lower and strictly upper triangular matrix $\tilde{A}=L+U$. The filter is then found by in each iteration $i$ solving the triangular system $L \tilde{f}^{(i)}=\tilde{b}-U \tilde{f}^{(i-1)}$. It can be shown that the iterates $\tilde{f}^{(i)}$ converge to the solution of the original problem $\tilde{A} \tilde{f}=\tilde{b}$ under the present assumptions [37].

By employing the Gauss-Seidel-based optimization, the SRDCF tracker introduced in Paper D achieves a tracking speed of few frames per second. While not yet real-time, it demonstrated superior robustness and accuracy compared to previous approaches, yet faster than many of its competitors. To further improve computational efficiency, and pave the way for the use of deep features, the main drawbacks of the Gauss-Seidel-based approach were addressed in Paper $\mathrm{H}$.

To optimize the more general loss derived in Paper $\mathrm{H}$, a Conjugate Gradient (CG) based strategy is proposed. CG can be applied to any set of normal equations $A \hat{f}=b$ of full rank. It is based on finding a set of conjugate directions $p^{(i)}$ and optimal step lengths $\beta^{(i)}$ used for updating the filter $\hat{f}^{(i)}=\hat{f}^{(i-1)}+\beta^{(i)} p^{(i)}$. Theoretically, the algorithm converges to the solution in a finite number of iterations $i$. In practice however, the algorithm is stopped after a fixed number of iterations or when the error has decreased to a satisfactory level. A complete description of CG is outside the scope of 
this thesis. The interested reader is referred to $[82,71]$ for a comprehensive description.

The computational bottleneck of $\mathrm{CG}$ is the evaluation of the matrixvector product $A p^{(i)}$ in each iteration $i$. This is in fact the key advantage of CG. Recall that Gauss-Seidel requires solving a triangular system in each iteration. On the contrary, CG can exploit the particular sparsity structure of $A$ in the computation $A p^{(i)}$. As discussed in the supplementary material of Paper H, $A p^{(i)}$ can implemented as a series of simple block-wise dense matrixvector products, convolutions and point-wise multiplications. Exploiting this remarkable property removes the need for explicitly constructing $A$ using sparse matrix libraries. This reduces the quadratic $\mathcal{O}\left(D^{2}\right)$ complexity in the feature dimension to linear $\mathcal{O}(D)$, a crucial improvement enabling tractable integration of high-dimensional deep features. Furthermore, the complexvalued Fourier representation can be directly utilized, avoiding the additional change-of-basis performed in Paper D, which reduces the sparsity.

The proposed CG-based optimization procedure is also a crucial component in the ECO tracker, proposed in Paper I. In this work, the procedure is generalized to the non-linear case of optimizing the factorized convolution operator, discussed in section 3.7. Moreover, the CG algorithm is further adapted to the online learning scenario by employing the PolakRibieŕe update rule instead of the traditional Fletcher-Reeves [82]. A detailed description of the optimization problem structure and the integration of $\mathrm{CG}$ is provided in Paper $\mathrm{H}$ and Paper I, including their supplementary materials. 



\section{4}

\section{IMAGE FEATURES}

Image features, or descriptors, is one of the most central topics in computer vision. It regards finding a suitable representation of the underlying image data that facilitates the task at hand. In the case of visual object tracking, we require a representation that simplifies the localization of the target. This is particularly important when applying linear discriminative models, such as the DCF, restricted to finding a linear decision boundary. Image features have the potential of increasing the effectiveness of linear models by nonlinearly mapping the image samples to a higher-dimensional space, increasing linear separability between the target and background. This chapter gives an introduction to image features employed and evaluated in this thesis.

\subsection{Invariance and Discriminative Power}

When selecting or constructing an image feature representation for visual tracking there are two important concepts to consider, namely invariance and discriminative power. As we will see, these two desired properties are partially contradicting. Hence, a suitable trade-off is required. The choice of features thus depend on the tracking application itself, as the need for a particular invariance or discriminating ability may be important or not. This thesis focuses on more general features applicable to a wide range of object tracking scenarios.

As described in section 3.2, feature extraction is seen as a mapping $x=$ $\phi(J)$ from the original image $J$ to a multi-dimensional feature map $x$. A feature mapping is said to be invariant to a particular transformation of the image $J$ if it does not affect the resulting features $x$. For example, a particular type of photometric invariance is the insensitivity to the image brightness or intensity. Formally, this is achieved if the feature mapping satisfies $\phi(a J)=\phi(J)$ for any scalar factor $a>0$. That is, any increase or decrease in the image brightness does not affect the extracted features $x$. 


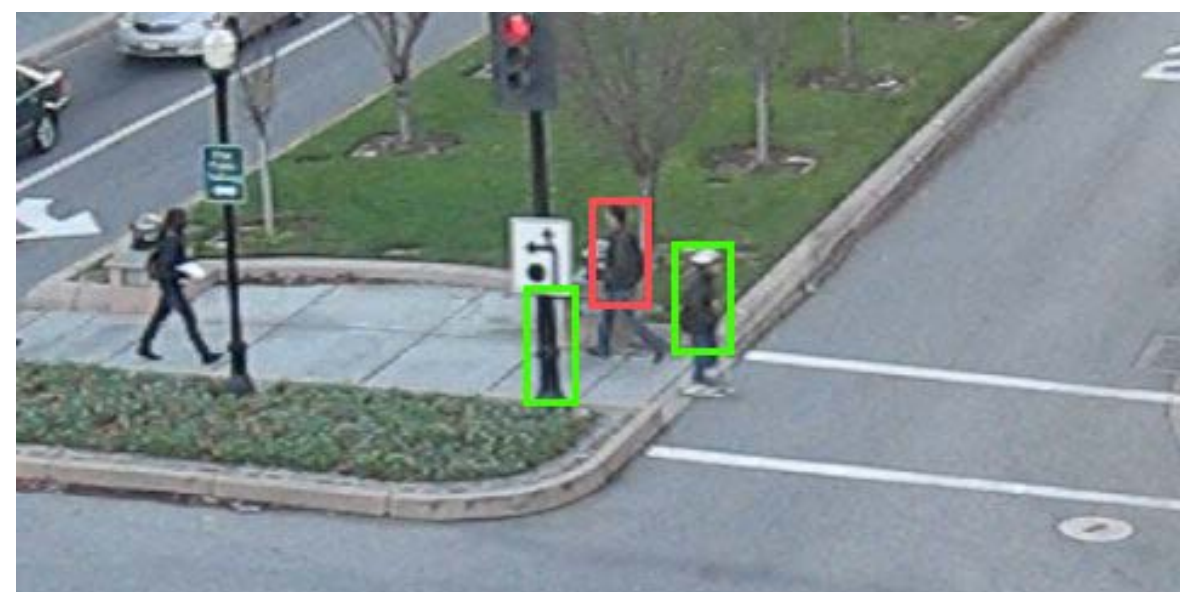

Figure 4.1: The target (red box) has similar color and shape as the two distractor objects (green boxes). Discriminative features are important to disambiguate such cases.

Such invariance is useful when tracking an object under varying illumination conditions. If the sun suddenly appears, illuminating the scene, our model should still be able to find the target as the representation $x$ of the image is practically unchanged. Features can also exhibit invariance on a higher semantic level. When tracking humans, features that are invariant to the human pose are of interest. Such invariance cannot be formalized as a simple low-level transformation of the image data $J$, as in the previous example. Instead, it relates to a more high-level concept.

Invariance is not the only desirable property of $\phi$. We can trivially construct an image representation that has full invariance to all possible transformations by simply setting $\phi(J)=0$. But this representation serves us no use as all information is destroyed. There are also target transformations, such as translation and scale, that we wish to estimate. If the representation were invariant to these transformations, they would remain unobservable in the feature map $x$. It is important to understand that there is a crucial distinction between the feature invariance discussed in this section and the spatial invariance defined in section 3.2. The latter meaning that a translation of the image $J$ should result in the corresponding translation of the feature map $x$. This form of invariance is referred to as equivariance in group theory.

Discriminative power is the more informal concept of how easily the target can be distinguished from the background using the given features. An example is given in figure 4.1. The target object, in this case the human in the red (center) box, has a color and shape very similar to the human to the right and the pole to the left, indicated by green boxes. Reliably disambiguating this case in the original RGB image space is therefore hard, in particular 

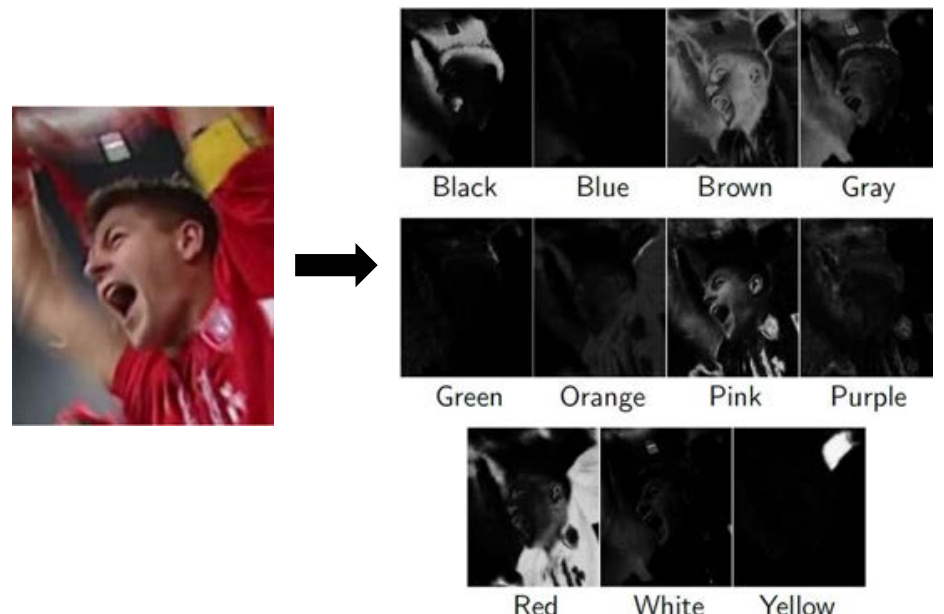

Figure 4.2: Visualization of the Color Names descriptor. Each RGB value is mapped to the probabilities of the 11 basic color terms in the English language.

when using a linear model. A feature mapping can unwrap the sample space, simplifying separability between the target and background. Discriminative features pronounces details or extracts higher-level information benefiting the model's ability to distinguish the target. It should be noted that some authors, e.g. [84] have a sightly different view of discriminative power, not including the ability to simplify discrimination.

\subsection{Color Features}

Color representations is a classical field of research dating back to the study of light and human perception of color [89]. Color spaces were proposed early on [8] to describe perceived colors and their relationship to colors produced by paint and illuminated displays. In image analysis and computer vision, color representations have been further developed for object recognition and other similar tasks. Paper A investigates a variety of color features for visual tracking. This analysis is further extended in Paper B by including soft histograms of low-dimensional color spaces. The experiments performed in both papers demonstrate the advantage of the Color Names representation [86] in terms of tracking performance, computation and compactness. It has therefore been employed in many of the subsequent papers by the author and is now widely used in the tracking community.

The Color Names descriptor was first used for tracking in Paper A. In general, Color Names are linguistic labels for representing colors in the world. The Color Names descriptor [86] is motivated by a linguistic study performed 


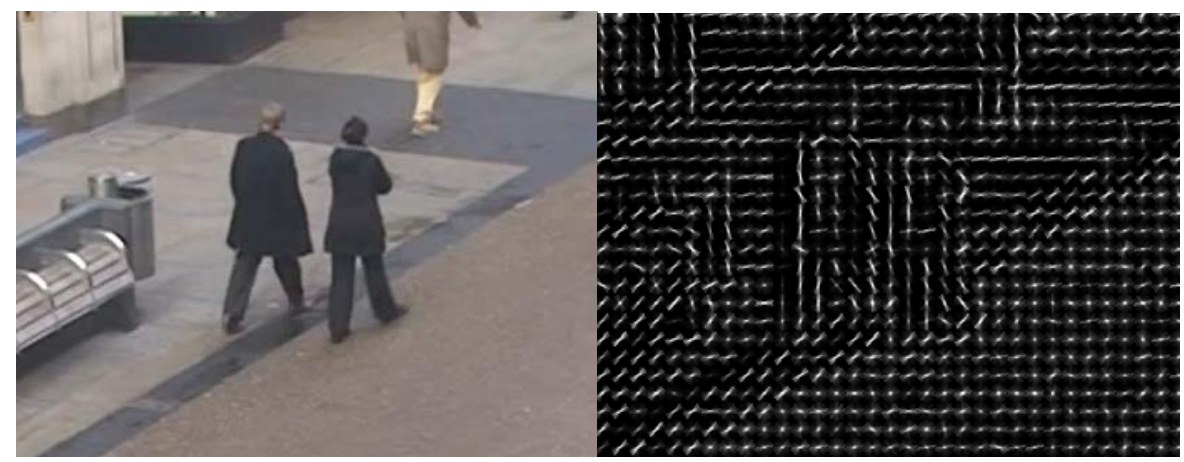

Figure 4.3: Visualization of the HOG feature map (right) extracted from the image (left). In each HOG cell, the magnitude of each gradient orientation is represented by the intensity of the line with the corresponding direction.

by Berlin and Kay [2], which concluded that the English language contains eleven basic color terms (see figure 4.2). In computer vision, color naming is the task of assigning a linguistic color label to an RGB observation. In the Color Names descriptor, this is performed by mapping an RGB value to a probabilistic representation of the 11 color terms. That is, the values in the descriptor represent the probabilities that the pixel should be assigned to the corresponding colors. The mapping was automatically learned by first collecting 100 images per color using Google Image search. Inspired by models in text analysis, probabilistic latent semantic analysis (PLSA) is then applied to learn the distribution of color values given a color term. During tracking, a fixed lookup table, provided by [86], from $32^{3}$ uniformly sampled RGB values to the 11 color name probabilities is used. This feature mapping is visualized in figure 4.2 .

\subsection{Histogram of Oriented Gradients}

Several of the papers in this thesis employ the Histogram of Oriented Gradients (HOG) feature introduced by Dalal and Triggs [9]. It was first used for human detection, and has since been commonly applied in object detection and visual tracking. Unlike color representations, HOG captures shape information by collecting statistics of the image gradients. It has therefore been highly successful for detecting pedestrians and other objects where the shape is particularly discriminative.

The HOG feature map is created by first computing the image gradient using simple derivative filters. Histograms of the gradient orientations are then formed in a dense image grid of cells, which are typically $8 \times 8$ pixels. Soft assignment with a linear function into nine bins is used to construct the histograms after the sign of the gradient has been discarded. The histogram 
vectors are then block normalized by dividing with the L2-norm in each of the four $2 \times 2$ cell neighborhoods. The result is a $4 \cdot 9=36$ dimensional descriptor at each cell. This descriptor was further refined by Felzenszwalb et al. [28], using a PCA-based dimensionality reduction resulting in a 31 dimensional feature map. Figure 4.3 visualizes the HOG feature map.

\subsection{Deep Features}

During the recent years deep learning has revolutionized many areas in computer vision, leading to extraordinary performance leaps in image classification $[7,40]$ and object detection [32, 77]. Deep Convolutional Neural Networks (CNNs) [36] have shown to be particularly well suited for image related tasks due to their spatial invariance properties. A CNN applies a sequence of convolutions and non-linear operations onto the image. The convolutions have trainable filters that are learned through back-propagation [80]. The non-linearities often consist of Rectified Linear Unit (ReLU) activations [35], max-pooling [36] and normalization [48]. When trained for classification, the final stages in the network usually consist of a linear fully-connected layer followed by a soft-max operation, which outputs the probabilities for each class. The network is then trained by minimizing a cross entropy loss using stochastic gradient descent [36].

Applying deep features for generic tracking has proved challenging. This is primarily due to the inherent scarcity of training data. Therefore, many tracking approaches employ deep CNNs that are pre-trained for the image classification task on, e.g., the ImageNet dataset [25]. Despite being trained for classification, such deep representations are versatile and applicable in a wide range of computer vision tasks [76]. In Paper E, a preliminary study of deep convolutional feature maps for tracking is performed. Convolutional layers from the popular VGG-M architecture [7] is used. The network had been pre-trained on the ImageNet dataset.

The analysis performed in the paper indicate the importance of shallowlayer features extracted from the network. Shallow layers contain low-level information at a high spatial resolution. Such features are important for accurate target localization, which is crucial in tracking to avoid model drift. On the other hand, deeper-layer feature maps possess high-level invariance to complex appearance changes, such as deformations and out-of-plane rotations. Thus, deeper layers have the potential of improving tracking robustness. However, the resolution of these layers is usually low due to pooling and strided convolutions. Moreover, the deeper layers tend to be invariant to small translation and scale changes, further reducing its localization abilities. Deep and shallow convolutional features are visualized in figure 4.4 (top row).

Paper $\mathrm{H}$ addresses the important question of how to fuse shallow and deep convolutional feature maps from a CNN. The aim is to exploit their 

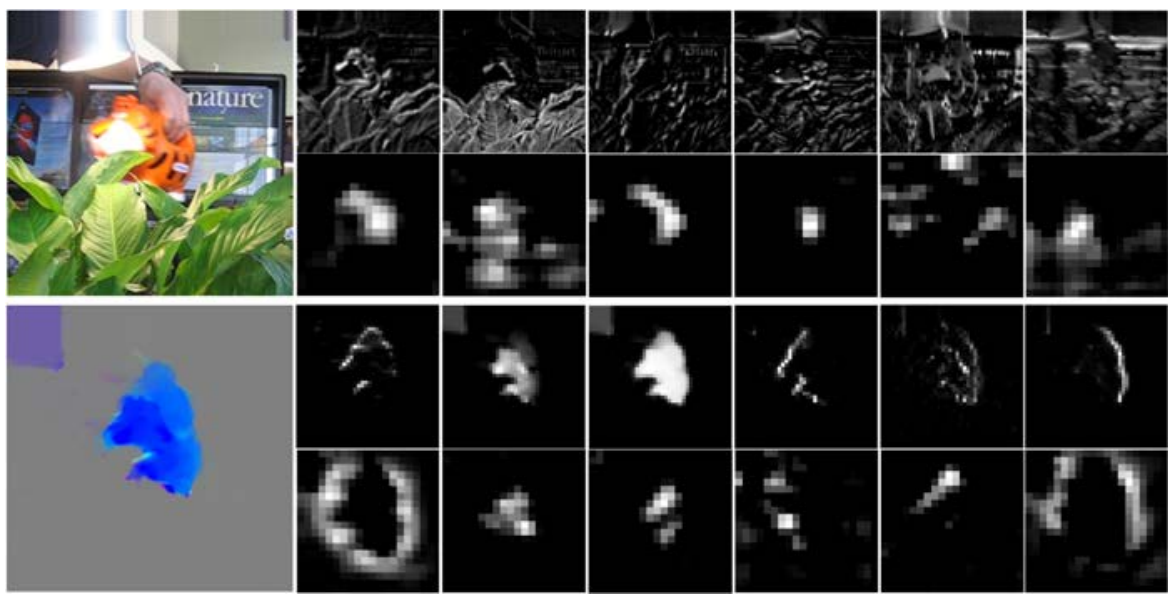

Figure 4.4: The top two rows visualize shallow and deep convolutional features extracted from the corresponding RGB image patch. The shallow features (top) capture low-level information, such as edges and color, while the deep activations represent high-level information. The bottom two rows visualize the corresponding layers from a motion network, taking the optical flow image (bottom left) as input.

complementary advantages for improved tracking accuracy and robustness. A continuous-domain formulation of the DCF framework is proposed that enables the integration of multi-resolution features. This formulation, named the Continuous Convolution Operator Tracker (C-COT), is introduced in section 3.6. With the proposed continuous-domain model, the C-COT can utilize shallow and deep convolutional layers, at different spatial resolutions, in a joint learning framework. Experiments performed in the paper clearly demonstrate the benefit of fusing deep and shallow convolutional features. Furthermore, C-COT achieved the top rank on the sequestered dataset in the most recent Visual Object Tracking (VOT) 2017 challenge [51]. Paper I investigates strategies to reduce the computational cost of the C-COT tracking framework and mitigates the risk of overfitting.

\subsection{Deep Motion Features}

All image features discussed so far in this chapter capture appearance information from a single image. Paper $\mathrm{G}$ goes beyond this restriction and investigates the use of deep motion features for tracking. The idea is to capture high-level information about the target motion and shape based on the optical flow between two frames. Optical flow is the dense motion field computed between two images. It carries information of the target motion as well as its shape. Deep motion features are extracted from a deep CNN taking 
optical flow images as input. Such features have previously been employed for action recognition [33], where the authors first trained a deep motion CNN for action classification. This motion network was employed in Paper G to investigate the impact of deep motion features for tracking.

Paper G analyzes deep appearance and motion features along with their combinations for visual tracking. The experiments strongly indicate that deep motion features are complementary to appearance features, benefiting tracking performance when combined with the latter. The best results are obtained when combining HOG, for accuracy, with deep convolutional features extracted from both the appearance and motion network. The deep motion features are visualized in figure 4.4 (bottom rows). 



\section{5}

\section{Concluding Remarks}

This thesis studies and advances the Discriminative Correlation Filter (DCF) framework for the task of generic visual tracking. Contributions are made in two main areas: (1) The DCF framework itself, i.e. appearance modeling, learning, inference and prediction. (2) Investigation and integration of sophisticated image features. This thesis demonstrates the importance of both areas in terms of final tracking performance and applicability.

Regarding the DCF framework (1), several key limitations and questions are addressed. The problem of how to tractably extend the DCF model to high-dimensional image feature representations is explored. Next, the issues introduced by circular convolution are addressed. Then a strategy is proposed to reduce the impact of faulty training samples collected during tracking. Furthermore, a theoretical framework for continuous-domain learning is developed, enabling the integration of multiresolution deep feature maps and sub-pixel prediction. To ensure practical applicability, dimensionality reduction techniques and efficient inference algorithms are proposed to reduce the computational footprint. Finally, the question of how to predict the scale of the target is investigated.

In addition to the aforementioned contributions to the DCF framework itself, the integration and impact of sophisticated image features for tracking (2) are explored. A thorough evaluation of a variety of color representations is performed. Then, the characteristics and impact of deep features for DCFbased tracking are investigated. Furthermore, a framework for fusing different convolutional layers is developed. Finally, the impact of deep motion features is investigated for visual tracking.

The advancements in visual tracking presented in this thesis have led to major performance improvements. The integration of color features in Paper A provides a relative gain of $26 \%$ in the area-under-the-curve (AUC) score [88], combining both accuracy and robustness. In Paper $\mathrm{C}$, the proposed scale estimation approach yields a relative $13 \%$ gain in AUC on the OTB-2013 
dataset [88]. The spatial regularization component and inference strategy proposed in Paper D further improves the result by $17 \%$ in AUC on the OTB-2015 dataset [87]. In Paper H, the integration of deep features into the continuous-domain learning formulation gives an additional $13 \%$ gain on the same dataset and reduced the relative failure rate on the VOT2015 dataset by $34 \%$.

The above indicators demonstrate the individual improvements originating from key contributions of this thesis. However, to obtain a broader view of the progress in the field of visual tracking and the impact of this thesis, the Visual Object Tracking (VOT) challenge results provide much valuable insight. Studying the official VOT2016 results [50], the first tracker published by the author, the ACT (Paper A), obtained an Expected Average Overlap (EAO) score of 0.173 . The ACT had achieved state-of-the-art results at time of publication 2014. Yet, the C-COT tracker published in Paper $\mathrm{H}$ the same year as the challenge, achieved top rank with an EAO score of 0.331, constituting an almost two-fold improvement. This shows the astonishing progress in visual tracking during the span of this thesis. In the latest version of the challenge: VOT2017 [51], all the top five trackers on the sequestered dataset directly built upon the C-COT or ECO framework, presented in Paper $\mathrm{H}$ and Paper I respectively, using the code published by the author.

Since the start of this thesis in 2014 the DCF framework has matured substantially. While not yet investigated in 2013, the use of multi-dimensional feature representations and scale estimation are now standard procedure. Similarly, the utilization of spatial regularization or similar strategies to alleviate periodic effects are now common. Furthermore, as demonstrated by the VOT2017 results [51], deep features are now well established and the use of iterative optimizers is becoming increasingly common. Despite these astounding improvements in recent years, object tracking is by no means a solved problem. There are multiple opportunities for future research.

The integration of deep learning techniques for visual tracking is still at an early stage. While it is an important factor in the recent progress, as demonstrated in Paper H and VOT2017 [51], their impact in the tracking field has been less compared to e.g. image classification and semantic segmentation. The lack of training data and computational restrictions pose major challenges to be addressed in future work, when incorporating deep representations for visual tracking. Further investigation and development of deep motion features is a related future line of research. Here, the immediate future goals are to exploit efficient networks and faster optical flow computation to ensure real-time performance.

From a more general perspective, there are many important and interesting research questions and topics to be addressed in visual tracking. The rapid advancement of short-term tracking techniques motivates a shift of focus towards long-term generic tracking. In this case, long-term robustness of the tracking model is of major importance. This is an open question in 
DCF-based tracking, where new model update and training set management strategies are of interest. One possible future research direction is along the joint learning approach proposed in Paper F. Another interesting direction is to train optimal update policies using reinforcement learning techniques [47].

Important future directions also include providing a richer tracking output. Most current approaches represent the target state as a bounding box. Future research should focus on extending this representation. One possibility is to estimate a pixel-wise segmentation mask of the target object. This brings interesting parallels with the field of video object segmentation [73] to be explored. In future, I therefore expect visual tracking evolve in new directions, while remaining an active and important research topic. 



\section{BIBLIOGRAPHY}

[1] Chenglong Bao, Yi Wu, Haibin Ling, and Hui Ji. "Real time robust L1 tracker using accelerated proximal gradient approach". In: IEEE Conference on Computer Vision and Pattern Recognition, Providence, RI, USA, June 16-21. 2012, pp. 1830-1837. DOI: 10.1109/CVPR. 2012. 6247881 .

[2] Brent Berlin and Paul Kay. Basic Color Terms: Their Universality and Evolution. Berkeley, CA: University of California Press, 1969.

[3] Luca Bertinetto, Jack Valmadre, Stuart Golodetz, Ondrej Miksik, and Philip H. S. Torr. "Staple: Complementary Learners for Real-Time Tracking". In: IEEE Conference on Computer Vision and Pattern Recognition, CVPR 2016, Las Vegas, NV, USA, June 27-30. 2016, pp. 1401-1409. DOI: 10.1109/CVPR.2016.156.

[4] Christopher M. Bishop. Pattern Recognition and Machine Learning (Information Science and Statistics). Secaucus, NJ, USA: SpringerVerlag New York, Inc., 2006. ISBN: 0387310738.

[5] David S. Bolme, J. Ross Beveridge, Bruce A. Draper, and Yui Man Lui. "Visual object tracking using adaptive correlation filters". In: The Twenty-Third IEEE Conference on Computer Vision and Pattern Recognition, CVPR, San Francisco, CA, USA, 13-18 June. 2010, pp. 2544-2550. DOI: 10.1109/CVPR . 2010.5539960.

[6] David Casasent. "Unified synthetic discriminant function computational formulation". In: Appl. Opt. 23.10 (May 1984), pp. 1620-1627. DOI: 10. 1364/A0.23.001620.

[7] Ken Chatfield, Karen Simonyan, Andrea Vedaldi, and Andrew Zisserman. "Return of the Devil in the Details: Delving Deep into Convolutional Nets". In: British Machine Vision Conference, BMVC, Nottingham, UK, September 1-5. 2014. URL: http://www. bmva.org/ bmvc/2014/papers/paper054/index.html. 
[8] CIE. Commission internationale de l'Eclairage proceedings, 1931. Cambridge: Cambridge University Press, 1932.

[9] Navneet Dalal and Bill Triggs. "Histograms of Oriented Gradients for Human Detection". In: IEEE Computer Society Conference on Computer Vision and Pattern Recognition CVPR, 20-26 June, San Diego, CA, USA. 2005, pp. 886-893. DOI: 10.1109/CVPR.2005.177.

[10] Martin Danelljan. "Visual Tracking". MA thesis. Linköping University, 2013. URL: http://urn.kb.se/resolve?urn=urn:nbn:se:liu:diva105659.

[11] Martin Danelljan, Goutam Bhat, Susanna Gladh, Fahad Shahbaz Khan, and Michael Felsberg. "Deep Motion and Appearance Cues for Visual Tracking". In: Pattern Recognition Letters (2018). DOI: 10 . 1016/ j . patrec.2018.03.009.

[12] Martin Danelljan, Goutam Bhat, Fahad Shahbaz Khan, and Michael Felsberg. "ECO: Efficient Convolution Operators for Tracking". In: IEEE Conference on Computer Vision and Pattern Recognition CVPR 2017, Honolulu, HI, USA, July 21-26. 2017, pp. 6931-6939. DOI: 10 . 1109/CVPR. 2017.733.

[13] Martin Danelljan, Gustav Häger, Fahad Shahbaz Khan, and Michael Felsberg. "Accurate Scale Estimation for Robust Visual Tracking". In: British Machine Vision Conference, BMVC 2014, Nottingham, UK, September 1-5. 2014. URL: http://www.bmva.org/bmvc/2014/papers/ paper038/index.html.

[14] Martin Danelljan, Gustav Häger, Fahad Shahbaz Khan, and Michael Felsberg. "Adaptive Decontamination of the Training Set: A Unified Formulation for Discriminative Visual Tracking". In: IEEE Conference on Computer Vision and Pattern Recognition, CVPR 2016, Las Vegas, NV, USA, June 27-30. 2016, pp. 1430-1438. DOI: 10.1109/CVPR. 2016. 159.

[15] Martin Danelljan, Gustav Häger, Fahad Shahbaz Khan, and Michael Felsberg. "Coloring Channel Representations for Visual Tracking". In: 19th Scandinavian Conference on Image Analysis, SCIA 2015, Copenhagen, Denmark, June 15-17. 2015, pp. 117-129. DOI: 10.1007/ 978-3-319-19665-7_10.

[16] Martin Danelljan, Gustav Häger, Fahad Shahbaz Khan, and Michael Felsberg. "Convolutional Features for Correlation Filter Based Visual Tracking". In: IEEE International Conference on Computer Vision ICCV Workshop 2015, Santiago, Chile, December 7-13. 2015, pp. 621629. DOI: 10.1109/ICCVW.2015.84. 
[17] Martin Danelljan, Gustav Häger, Fahad Shahbaz Khan, and Michael Felsberg. "Discriminative Scale Space Tracking". In: IEEE Transactions on Pattern Analysis and Machine Intelligence 39.8 (2017), pp. 15611575. DOI: 10.1109/TPAMI . 2016. 2609928.

[18] Martin Danelljan, Gustav Häger, Fahad Shahbaz Khan, and Michael Felsberg. "Learning Spatially Regularized Correlation Filters for Visual Tracking". In: IEEE International Conference on Computer Vision, ICCV 2015, Santiago, Chile, December 7-13. 2015, pp. 4310-4318. DOI: 10.1109/ICCV.2015.490.

[19] Martin Danelljan, Fahad Shahbaz Khan, Michael Felsberg, Karl Granström, Fredrik Heintz, Piotr Rudol, Mariusz Wzorek, Jonas Kvarnström, and Patrick Doherty. "A Low-Level Active Vision Framework for Collaborative Unmanned Aircraft Systems". In: European Conference on Computer Vision ECCV Workshops 2016 Zurich, Switzerland, September 6-7 and 12. 2014, pp. 223-237. DOI: 10.1007/978-3-319-16178-5_15.

[20] Martin Danelljan, Fahad Shahbaz Khan, Michael Felsberg, and Joost van de Weijer. "Adaptive Color Attributes for Real-Time Visual Tracking". In: IEEE Conference on Computer Vision and Pattern Recognition, CVPR 2014, Columbus, OH, USA, June 23-28. 2014, pp. 1090-1097. DOI: 10.1109/CVPR.2014.143.

[21] Martin Danelljan, Giulia Meneghetti, Fahad Shahbaz Khan, and Michael Felsberg. "A Probabilistic Framework for Color-Based Point Set Registration". In: IEEE Conference on Computer Vision and Pattern Recognition, CVPR 2016, Las Vegas, NV, USA, June 27-30. 2016, pp. 1818-1826. DOI: 10.1109/CVPR.2016.201.

[22] Martin Danelljan, Giulia Meneghetti, Fahad Shahbaz Khan, and Michael Felsberg. "Aligning the dissimilar: A probabilistic method for feature-based point set registration". In: 23rd International Conference on Pattern Recognition, ICPR 2016, Cancún, Mexico, December 4-8. 2016, pp. 247-252. DOI: 10.1109/ICPR.2016.7899641.

[23] Martin Danelljan, Andreas Robinson, Fahad Shahbaz Khan, and Michael Felsberg. "Beyond Correlation Filters: Learning Continuous Convolution Operators for Visual Tracking". In: 14th European Conference on Computer Vision ECCV, Amsterdam, The Netherlands, October 11-14, 2016, Proceedings, Part V. 2016, pp. 472-488. DOI: 10. 1007/978-3-319-46454-1_29.

[24] E. De Castro and C. Morandi. "Registration of Translated and Rotated Images Using Finite Fourier Transforms". In: IEEE Trans. Pattern Anal. Mach. Intell. 9.5 (May 1987), pp. 700-703. ISSN: 0162-8828. DOI: 10. 1109/TPAMI . 1987.4767966. 
[25] Jia Deng, Wei Dong, Richard Socher, Li-Jia Li, Kai Li, and Fei-Fei Li. "ImageNet: A large-scale hierarchical image database". In: 2009 IEEE Computer Society Conference on Computer Vision and Pattern Recognition CVPR, 20-25 June, Miami, Florida, USA. 2009, pp. 248255. DOI: $10.1109 / C V P R W .2009 .5206848$.

[26] Michael Felsberg, Amanda Berg, Gustav Häger, Jörgen Ahlberg, Matej Kristan, Jiri Matas, Ales Leonardis, Luka Cehovin, Gustavo Fernández, Tomás Vojír, Georg Nebehay, and Roman P. Pflugfelder. "The Thermal Infrared Visual Object Tracking VOT-TIR2015 Challenge Results". In: IEEE International Conference on Computer Vision Workshop, ICCV Workshops, Santiago, Chile, December 7-13. 2015, pp. 639-651. DoI: 10.1109/ICCVW. 2015.86.

[27] Michael Felsberg, Per-Erik Forssén, and Hanno Scharr. "Channel Smoothing: Efficient Robust Smoothing of Low-Level Signal Features". In: IEEE Transactions on Pattern Analysis and Machine Intelligence 28.2 (2006), pp. 209-222. DOI: 10.1109/TPAMI.2006.29.

[28] Pedro F. Felzenszwalb, Ross B. Girshick, David A. McAllester, and Deva Ramanan. "Object Detection with Discriminatively Trained PartBased Models". In: IEEE Transactions on Pattern Analysis Machine Intelligence 32.9 (2010), pp. 1627-1645. DOI: 10.1109/TPAMI . 2009.167.

[29] Hamed Kiani Galoogahi, Terence Sim, and Simon Lucey. "Multi-channel Correlation Filters". In: IEEE International Conference on Computer Vision, ICCV, Sydney, Australia, December 1-8. 2013, pp. 3072-3079. DOI: $10.1109 /$ ICCV. 2013.381.

[30] Changxin Gao, Feifei Chen, Jin-Gang Yu, Rui Huang, and Nong Sang. "Exemplar-based linear discriminant analysis for robust object tracking". In: IEEE International Conference onImage Processing (ICIP). 2014, pp. 388-392.

[31] Claude Gasquet and Patrick Witomski. Fourier Analysis and Applications: Filtering, Numerical Computation, Wavelets. Texts in Applied Mathematics. Springer-Verlag New York Inc., 1999. ISBN: 0387-98485-2.

[32] Ross B. Girshick, Jeff Donahue, Trevor Darrell, and Jitendra Malik. "Rich Feature Hierarchies for Accurate Object Detection and Semantic Segmentation". In: IEEE Conference on Computer Vision and Pattern Recognition, CVPR, Columbus, OH, USA, June 23-28. 2014, pp. 580587. DOI: 10.1109/CVPR. 2014.81.

[33] Georgia Gkioxari and Jitendra Malik. "Finding action tubes". In: IEEE Conference on Computer Vision and Pattern Recognition, CVPR, Boston, MA, USA, June 7-12. 2015, pp. 759-768. DOI: 10.1109/CVPR. 2015.7298676 . 
[34] Susanna Gladh, Martin Danelljan, Fahad Shahbaz Khan, and Michael Felsberg. "Deep motion features for visual tracking". In: 23rd International Conference on Pattern Recognition, ICPR 2016, Cancún, Mexico, December 4-8. 2016, pp. 1243-1248. DOI: 10.1109/ICPR. 2016. 7899807.

[35] Xavier Glorot, Antoine Bordes, and Yoshua Bengio. "Deep Sparse Rectifier Neural Networks". In: Proceedings of the Fourteenth International Conference on Artificial Intelligence and Statistics, AISTATS 2011, Fort Lauderdale, USA, April 11-13, 2011. 2011, pp. 315-323. URL: http://www.jmlr.org/proceedings/papers/v15/ glorot11a/glorot11a.pdf.

[36] Ian Goodfellow, Yoshua Bengio, and Aaron Courville. Deep Learning. MIT Press, 2016. URL: http://www. deeplearningbook.org.

[37] A Hadjidimos. "Successive overrelaxation (SOR) and related methods". In: 123 (Nov. 2000), pp. 177-199.

[38] Gustav Häger, Goutam Bhat, Martin Danelljan, Fahad Shahbaz Khan, Michael Felsberg, Piotr Rudol, and Patrick Doherty. "Combining Visual Tracking and Person Detection for Long Term Tracking on a UAV". In: 12th International Symposium on Visual Computing, ISVC 2016, Las Vegas, NV, USA, December 12-14. 2016, pp. 557-568. DOI: $10.1007 /$ 978-3-319-50835-1_50.

[39] R. I. Hartley and A. Zisserman. Multiple View Geometry in Computer Vision. Second. Cambridge University Press, 2004. ISBN: 0521540518.

[40] Kaiming He, Xiangyu Zhang, Shaoqing Ren, and Jian Sun. "Deep Residual Learning for Image Recognition". In: IEEE Conference on Computer Vision and Pattern Recognition, CVPR, Las Vegas, NV, USA, June 27-30. 2016. DOI: 10.1109/CVPR.2016.90.

[41] João F. Henriques, Rui Caseiro, Pedro Martins, and Jorge Batista. "High-Speed Tracking with Kernelized Correlation Filters". In: IEEE Transactions on Pattern Analysis and Machine Intelligence 37.3 (2015), pp. 583-596. DOI: 10.1109/TPAMI.2014.2345390.

[42] João F. Henriques, Rui Caseiro, Pedro Martins, and Jorge P. Batista. "Exploiting the Circulant Structure of Tracking-by-Detection with Kernels". In: 12th European Conference on Computer Vision ECCV, Florence, Italy, October 7-13, 2012, Proceedings, Part IV. 2012, pp. 702715. DOI: $10.1007 / 978-3-642-33765-9 \_50$.

[43] Charles F. Hester and David Casasent. "Multivariant technique for multiclass pattern recognition". In: Appl. Opt. 19.11 (June 1980), pp. 1758-1761. DOI: 10.1364/A0.19.001758. 
[44] Zhibin Hong, Zhe Chen, Chaohui Wang, Xue Mei, Danil V. Prokhorov, and Dacheng Tao. "MUlti-Store Tracker (MUSTer): A cognitive psychology inspired approach to object tracking". In: IEEE Conference on Computer Vision and Pattern Recognition, CVPR, Boston, MA, USA, June 7-12. 2015, pp. 749-758. DOI: 10.1109/CVPR.2015.7298675.

[45] Roger A. Horn and Charles R. Johnson. Matrix Analysis. 2nd. New York, NY, USA: Cambridge University Press, 2012.

[46] Joseph L. Horner and Peter D. Gianino. "Phase-only matched filtering". In: Appl. Opt. 23.6 (Mar. 1984), pp. 812-816. DOI: 10.1364/A0. 23 . 000812.

[47] James Steven Supancic III and Deva Ramanan. "Tracking as Online Decision-Making: Learning a Policy from Streaming Videos with Reinforcement Learning". In: IEEE International Conference on Computer Vision, ICCV 2017, Venice, Italy, October 22-29. 2017, pp. 322-331. DOI: 10.1109/ICCV.2017.43.

[48] Sergey Ioffe and Christian Szegedy. "Batch Normalization: Accelerating Deep Network Training by Reducing Internal Covariate Shift". In: Proceedings of the 32nd International Conference on Machine Learning, ICML, Lille, France, 6-11 July. 2015, pp. 448-456. URL: http://jmlr. org/proceedings/papers/v37/ioffe15.html.

[49] Joakim Johnander, Martin Danelljan, Fahad Shahbaz Khan, and Michael Felsberg. "DCCO: Towards Deformable Continuous Convolution Operators for Visual Tracking". In: 17th International Conference on Computer Analysis of Images and Patterns, CAIP 2017, Ystad, Sweden, August 22-24. 2017, pp. 55-67. DOI: 10.1007/978-3319-64689-3_5.

[50] Matej Kristan, Ales Leonardis, Jiri Matas, Michael Felsberg, Roman P. Pflugfelder, Luka Cehovin, Tomás Vojír, Gustav Häger, Alan Lukezic, Gustavo Fernández, and et al. "The Visual Object Tracking VOT2016 Challenge Results". In: European Conference on Computer Vision ECCV Workshops - Amsterdam, The Netherlands, October 8-10 and 15-16, Proceedings, Part II. 2016, pp. 777-823. DOI: 10.1007/978-3319-48881-3_54.

[51] Matej Kristan, Ales Leonardis, Jiri Matas, Michael Felsberg, Roman P. Pflugfelder, Luka Cehovin Zajc, Tomas Vojir, Gustav Häger, Alan Lukezic, Abdelrahman Eldesokey, Gustavo Fernández, and et al. "The Visual Object Tracking VOT2017 Challenge Results". In: IEEE International Conference on Computer Vision Workshops, ICCV Workshops, Venice, Italy, October 22-29. 2017, pp. 1949-1972. DOI: 10. 1109/ICCVW. 2017. 230. 
[52] Matej Kristan, Jiri Matas, Ales Leonardis, Michael Felsberg, Luka Cehovin, Gustavo Fernández, Tomás Vojír, Gustav Häger, Georg Nebehay, Roman P. Pflugfelder, and et al. "The Visual Object Tracking VOT2015 Challenge Results". In: IEEE International Conference on Computer Vision Workshop, ICCV Workshops, Santiago, Chile, December 7-13. 2015, pp. 564-586. DOI: 10.1109/ICCVW.2015.79.

[53] Matej Kristan, R. Pflugfelder, Ales Leonardis, Jiri Matas, Fatih Porikli, Luka Cehovin, Georg Nebehay, Fernandez Gustavo, and Tomas Vojir. "The Visual Object Tracking VOT2013 challenge results". In: IEEE International Conference on Computer Vision Workshop, ICCV Workshops. 2013, pp. 98-111.

[54] Matej Kristan, Roman P. Pflugfelder, Ales Leonardis, Jiri Matas, Luka Cehovin, Georg Nebehay, Tomás Vojír, Gustavo Fernández, and et al. "The Visual Object Tracking VOT2014 Challenge Results". In: European Conference on Computer Vision - ECCV Workshops - Zurich, Switzerland, September 6-7 and 12, Proceedings, Part II. 2014, pp. 191217. DOI: $10.1007 / 978-3-319-16181-5 \_14$.

[55] C. D. Kuglin and D. C. Hines. "The phase correlation image alignment method". In: IEEE Conference on Cybernetics and Society (1975), pp. $163-165$.

[56] B. V. K. Vijaya Kumar. "Minimum-variance synthetic discriminant functions". In: J. Opt. Soc. Am. A 3.10 (Oct. 1986), pp. 1579-1584. DOI: $10.1364 /$ JOSAA.3.001579.

[57] Bhagavatula Vijaya Kumar, Abhijit Mahalanobis, Sewoong Song, S. Richard F. Sims, and Jim F. Epperson. "Minimum squared error synthetic discriminant functions". In: Optical Engineering 31 (1992), p. 915 . DOI: $10.1117 / 12.56169$.

[58] Felix Järemo Lawin, Martin Danelljan, Fahad Shahbaz Khan, Per-Erik Forssén, and Michael Felsberg. "A Probabilistic Framework for ColorBased Point Set Registration". In: IEEE Conference on Computer Vision and Pattern Recognition, CVPR 2018, Salt Lake City, UT, USA, June 18-22. 2018.

[59] Felix Järemo Lawin, Martin Danelljan, Patrik Tosteberg, Goutam Bhat, Fahad Shahbaz Khan, and Michael Felsberg. "Deep Projective 3D Semantic Segmentation". In: 17th International Conference on Computer Analysis of Images and Patterns, CAIP 2017, Ystad, Sweden, August 22-24. 2017, pp. 95-107. DOI: 10.1007/978-3-319-64689-3_8.

[60] Pengpeng Liang, Erik Blasch, and Haibin Ling. "Encoding Color Information for Visual Tracking: Algorithms and Benchmark". In: IEEE Transactions on Image Processing 24.12 (2015), pp. 5630-5644. DOI: 10.1109/TIP. 2015.2482905. 
[61] C. C. Lin, S. H. Yen, and C. T. Tu. "Visual object tracking via LDA". In: 2017 International Conference on Applied System Innovation (ICASI). May 2017, pp. 315-318. DOI: 10.1109/ICASI.2017.7988415.

[62] Baiyang Liu, Junzhou Huang, Lin Yang, and Casimir A. Kulikowski. "Robust tracking using local sparse appearance model and K-selection". In: IEEE Conference on Computer Vision and Pattern Recognition, CVPR, Colorado Springs, CO, USA, 20-25 June. 2011, pp. 1313-1320. DOI: $10.1109 / C V P R .2011 .5995730$.

[63] Bruce D. Lucas and Takeo Kanade. "An Iterative Image Registration Technique with an Application to Stereo Vision". In: Proceedings of the 7th International Joint Conference on Artificial Intelligence, IJCAI, Vancouver, BC, Canada, August 24-28. 1981, pp. 674-679.

[64] Alan Lukežič, Tom'aš Voj'iř, Luka Čehovin Zajc, Jiř'i Matas, and Matej Kristan. "Discriminative Correlation Filter Tracker with Channel and Spatial Reliability". In: International Journal of Computer Vision (2018).

[65] Chao Ma, Xiaokang Yang, Chongyang Zhang, and Ming-Hsuan Yang. "Long-term correlation tracking". In: IEEE Conference on Computer Vision and Pattern Recognition, CVPR, Boston, MA, USA, June 7-12. 2015, pp. 5388-5396. DOI: 10.1109/CVPR.2015.7299177.

[66] Abhijit Mahalanobis, B. V. K. Vijaya Kumar, and David Casasent. "Minimum average correlation energy filters". In: Appl. Opt. 26.17 (Sept. 1987), pp. 3633-3640. DOI: 10.1364/A0.26.003633.

[67] Abhijit Mahalanobis, B. V. K. Vijaya Kumar, Sewoong Song, S. R. F. Sims, and J. F. Epperson. "Unconstrained correlation filters". In: Appl. Opt. 33.17 (June 1994), pp. 3751-3759. DOI: 10.1364/A0.33.003751.

[68] Giulia Meneghetti, Martin Danelljan, Michael Felsberg, and Klas Nordberg. "Image Alignment for Panorama Stitching in Sparsely Structured Environments". In: 19th Scandinavian Conference, SCIA 2015, Copenhagen, Denmark, June 15-17. 2015, pp. 428-439. DOI: 10. 1007/978-3-319-19665-7_36.

[69] Matthias Mueller, Neil Smith, and Bernard Ghanem. "A Benchmark and Simulator for UAV Tracking". In: 14th European Conference Computer Vision - ECCV, Amsterdam, The Netherlands, October 1114, Proceedings, Part I. 2016, pp. 445-461. DOI: 10.1007/978-3-31946448-0_27.

[70] Kevin P. Murphy. Machine Learning: A Probabilistic Perspective. The MIT Press, 2012. ISBN: 0262018020.

[71] J. Nocedal and S. J. Wright. Numerical Optimization. 2nd. Springer, 2006. 
[72] OpenCV. The OpenCV State of the Art Vision Challenge. http://code. opencv . org / projects / opencv/wiki / VisionChallenge. Accessed: 2015-09-17.

[73] Federico Perazzi, Jordi Pont-Tuset, Brian McWilliams, Luc J. Van Gool, Markus H. Gross, and Alexander Sorkine-Hornung. "A Benchmark Dataset and Evaluation Methodology for Video Object Segmentation". In: IEEE Conference on Computer Vision and Pattern Recognition, CVPR 2016, Las Vegas, NV, USA, June 27-30. 2016, pp. 724-732. DOI: 10.1109/CVPR. 2016.85.

[74] Lei Qu, Guoqiang Zhao, Baochen Yao, and Yuzhen Li. "Visual tracking with genetic algorithm augmented logistic regression". In: Signal, Image and Video Processing 12.1 (Jan. 2018), pp. 33-40. ISSN: 1863-1711. DOI: 10.1007/s11760-017-1127-2.

[75] Carl Edward Rasmussen and Christopher K. I. Williams. Gaussian Processes for Machine Learning (Adaptive Computation and Machine Learning). The MIT Press, 2005. ISBN: 026218253X.

[76] Ali Sharif Razavian, Hossein Azizpour, Josephine Sullivan, and Stefan Carlsson. "CNN Features Off-the-Shelf: An Astounding Baseline for Recognition". In: IEEE Conference on Computer Vision and Pattern Recognition, CVPR Workshops 2014, Columbus, OH, USA, June 2328, 2014. 2014. DOI: 10.1109/CVPRW.2014.131.

[77] Joseph Redmon and Ali Farhadi. "YOLO9000: Better, Faster, Stronger". In: 2017 IEEE Conference on Computer Vision and Pattern Recognition, CVPR 2017, Honolulu, HI, USA, July 21-26, 2017. 2017. DOI: 10.1109/ CVPR. 2017.690.

[78] Ph. Refregier. "Optimal trade-off filters for noise robustness, sharpness of the correlation peak, and Horner efficiency". In: Opt. Lett. 16.11 (June 1991), pp. 829-831. DOI: 10.1364/OL.16.000829.

[79] David A. Ross, Jongwoo Lim, Ruei-Sung Lin, and Ming-Hsuan Yang. "Incremental Learning for Robust Visual Tracking". In: International Journal of Computer Vision 77.1-3 (2008), pp. 125-141. DOI: 10.1007/ s11263-007-0075-7.

[80] David E. Rumelhart, Geoffrey E. Hinton, and Ronald J. Williams. "Learning representations by back-propagating errors". In: Nature 323.6088 (Oct. 1986), pp. 533-536. URL: http://dx . doi .org/10 . 1038/323533a0.

[81] Laura Sevilla-Lara and Erik G. Learned-Miller. "Distribution fields for tracking". In: IEEE Conference on Computer Vision and Pattern Recognition, Providence, RI, USA, June 16-21. 2012, pp. 1910-1917. DOI: $10.1109 /$ CVPR. 2012.6247891. 
[82] Jonathan R Shewchuk. An Introduction to the Conjugate Gradient Method Without the Agonizing Pain. Tech. rep. Pittsburgh, PA, USA, 1994.

[83] Arnold Smeulders, Dung Manh Chu, Rita Cucchiara, Simone Calderara, Afshin Dehghan, and Mubarak Shah. "Visual Tracking: An Experimental Survey". In: IEEE Transactions on Pattern Analysis and Machine Intelligence 36.7 (2014), pp. 1442-1468. DOI: 10.1109/TPAMI. 2013. 230.

[84] Manik Varma and Debajyoti Ray. "Learning The Discriminative PowerInvariance Trade-Off". In: IEEE 11th International Conference on Computer Vision, ICCV, Rio de Janeiro, Brazil, October 14-20. 2007. DOI: 10.1109/ICCV . 2007.4408875.

[85] H. Wang and F. Wang. "Visual tracking using logistic regression and sparse representation". In: 2014 7th International Congress on Image and Signal Processing. Oct. 2014, pp. 66-72. DOI: 10.1109/CISP. 2014. 7003751.

[86] Joost van de Weijer, Cordelia Schmid, Jakob J. Verbeek, and Diane Larlus. "Learning Color Names for Real-World Applications". In: IEEE Transactions on Image Processing 18.7 (2009), pp. 1512-1523. DOI: 10. 1109/TIP. 2009. 2019809.

[87] Yi Wu, Jongwoo Lim, and Ming-Hsuan Yang. "Object Tracking Benchmark". In: IEEE Transacitons on Pattern Analysis and Machine Intelligence 37.9 (2015), pp. 1834-1848. DOI: 10.1109/TPAMI . 2014. 2388226.

[88] Yi Wu, Jongwoo Lim, and Ming-Hsuan Yang. "Online Object Tracking: A Benchmark". In: IEEE Conference on Computer Vision and Pattern Recognition, Portland, OR, USA, June 23-28. 2013, pp. 2411-2418. DOI: 10.1109/CVPR. 2013.312.

[89] Thomas Young. "II. The Bakerian Lecture. On the theory of light and colours". In: Philosophical Transactions of the Royal Society of London 92 (1802), pp. 12-48. DOI: 10.1098/rstl.1802.0004.

[90] Hengshuang Zhao, Jianping Shi, Xiaojuan Qi, Xiaogang Wang, and Jiaya Jia. "Pyramid Scene Parsing Network". In: IEEE Conference on Computer Vision and Pattern Recognition, CVPR 2017, Honolulu, HI, USA, July 21-26. 2017, pp. 6230-6239. DOI: 10.1109/CVPR.2017.660. 
PART II

Publications 


\section{Publications}

The papers associated with this thesis have been removed for copyright reasons. For more details about these see:

http://urn.kb.se/resolve?urn=urn:nbn:se:liu:diva-147543 